\title{
Enhanced stability of superparamagnetic iron oxide nanoparticles in biological media using a pH adjusted-BSA adsorption protocol
}

\author{
Si-Ming Yu, Anna Laromaine*, Anna Roig* \\ Institut Ciència de Materials de Barcelona, Campus UAB, 08193 Bellaterra, Spain
}

\begin{abstract}
Superparamagnetic iron oxide nanoparticles (SPIONs) are widely used for biological applications due to their unique properties compared to their bulk counterparts, simplified SPIONs stabilization protocols applicable for a wide spectra of biological media remains a challenging issue. In this work, SPIONs with different surface coatings, tetramethylammonium hydroxide coated SPIONs (T-SPIONs) and citrate coated SPIONs (C-SPIONs), were synthesized by a facile, rapid and cost effective microwave assisted method. CSPIONs show robust stability in biological media of PBS and RPMI, while destabilize in DMEM. T-SPIONs were found to aggregate rapidly and significantly in all tested media. Then, a modified $\mathrm{pH}$ adjusted-BSA adsorption protocol and an addition of excess trisodium citrate dihydrate $\left(\mathrm{Na}_{3} \mathrm{Cit}\right)$ were used to enhance their stability in the media. The BSA adsorption protocol showed great efficiency in stabilizing the dispersed state of both SPIONs in the tested media, while the addition of excess $\mathrm{Na}_{3} \mathrm{Cit}$ showed limit effect, and it was only applicable for C-SPIONs. The formed BSA layer on SPIONs could be imaged by negative staining TEM, and revealed by Cryo-TEM, FTIR, DLS and the zeta potential measurements. Results indicated that BSA forms a monolayer of a thickness of about $3 \pm 1 \mathrm{~nm}$ and BSA interacts with C-SPIONs and T-SPIONs through the coating of the SPIONs, rather than by replacing them and interacting directly with the SPIONs surface. This synthetic method and stabilization protocol offer a general methodology to obtain SPIONs with a variety of surfactants, stable in different biological media in few minutes.
\end{abstract}

Keywords Microwave synthesis, iron oxide nanoparticles, biological media, stability, bovine serum albumin, citrate

*Corresponding authors: Anna Laromaine and Anna Roig; e-mail: alaromaine@icmab.es, roig@icmab.es, www.icmab.es/nn; Postal address: Institut de Ciència de Materials de Barcelona (ICMAB-CSIC), Campus de la UAB, 08193 Bellaterra (Barcelona), SPAIN; Tel.: + 34935801853 


\section{Abbreviations}

BSA Bovine serum albumin

BSA- T-SPIONs T-SPIONs after BSA adsorption

BSA- C-SPIONs C-SPIONs after BSA adsorption

C-SPIONs Superparamagnetic iron oxide nanoparticles coated with $\mathrm{Na}_{3} \mathrm{Cit}$

DMEM Dulbecco's Modified Eagle's Medium

DMEM- $\mathrm{Na}_{3} \mathrm{Cit} \quad$ DMEM with $10 \mathrm{mM} \mathrm{Na}_{3} \mathrm{Cit}$

DMEM-FBS DMEM with $10 \%$ FBS

DMEM-FBS- $\mathrm{Na}_{3}$ Cit DMEM with $10 \% \mathrm{FBS}$ and $10 \mathrm{mM} \mathrm{Na}_{3} \mathrm{Cit}$

FBS Fetal Bovine Serum

$\mathrm{Na}_{3} \mathrm{Cit} \quad$ Trisodium citrate dihydrate

PBS Phosphate buffered saline

PBS- $\mathrm{Na}_{3} \mathrm{Cit} \quad$ PBS with $10 \mathrm{mM} \mathrm{Na}_{3} \mathrm{Cit}$

RPMI Roswell Park Memorial Institute Medium

RPMI- $\mathrm{Na}_{3} \mathrm{Cit} \quad$ RPMI with $10 \mathrm{mM} \mathrm{Na}_{3} \mathrm{Cit}$

RPMI-FBS RPMI with $10 \%$ FBS

RPMI-FBS- $\mathrm{Na}_{3} \mathrm{Cit} \quad$ RPMI with $10 \% \mathrm{FBS}$ and $10 \mathrm{mM} \mathrm{Na}_{3} \mathrm{Cit}$

SPIONs Superparamagnetic iron oxide nanoparticles

TMAOH Tetramethylammonium hydroxide

T-SPIONs Superparamagnetic iron oxide nanoparticles coated with TMAOH 


\section{Introduction}

In recent years, the rapid development of modern nanotechnology emerged various nanoparticles (NPs) for biomedical applications. Among these NPs, superparamagnetic iron oxide NPs (SPIONs) are extensively investigated. Besides the properties of ultra-small size and high surface area to volume ratio, their unique magnetic properties, particular surface reactivity and excellent biocompatibility have paved the way for SPIONs to be effectively applied in biomedicine. Some of their biomedical applications are drug and gene delivery, hyperthermia therapy, magnetic resonance imaging and cell separation(Grief and Richardson 2005; McBain et al. 2008; Hergt and Dutz 2007; Carenza et al. 2014a; Gupta and Gupta 2005; Pamme and Wilhelm 2006; Levy et al. 2011; Di Corato et al. 2009; Figuerola et al. 2008; Corti et al. 2008b).

To date, many methods have been developed to synthesize SPIONs, including co-precipitation (Wei et al. 2011), thermal decomposition (Sun and Zeng 2002), microemulsion(Deng et al. 2003), and microwave assisted synthesis (Baghbanzadeh et al. 2011). Co-precipitation method is generally easy and affords high yields, but the reported SPIONs are rather polydisperse and their crystallinity is poor (Lu et al. 2007). Although both polydispersity and crystallinity can be improved by thermal decomposition, SPIONs synthesized by this method are usually hydrophobic and sometimes with a toxic surface coating which hampers their applications in bio-related fields. The yield in microemulsion method is the lowest compared to other methods, and also it requires large amount of solvents, which indicates it is a poor efficient method (Lu et al. 2007). Recently, microwave assisted synthesis has therefore become the interest of many researchers due to its rather simple synthetic process, homogeneity of the temperature and high yields within short time. Moreover, SPIONs synthesized by this method usually show a high saturation magnetization value and low surface reactivity. (Pascu et al. 2012).

Biomedical applications of SPIONs, or NPs in general, involve inevitably the contact with biological fluids, which are media with high ionic strength and/or various biomolecules. One main issue that researchers face is the stability of NPs in those complex biological media. Due to the interaction with the biological media, NPs tend to destabilize and undergo to aggregation processes, leading to the significant increase in their hydrodynamic size, as well as changes in NPs surface properties in terms of shape, surface area and charge. (Pratten and Lloyd 1986; Sager et al. 2007; Ji et al. 2010; Metin et al. 2011; Safi et al. 2010). Recent studies also evidenced that some biological responses greatly depend on the behavior of NPs in the biological media, the change in NPs hydrodynamic size probably influence their final bio-distribution and trigger desirable or deleterious biological responses. (Safi et al. 2010; Safi et al. 2011; Petri-Fink et al. 2008; Butoescu et al. 2009; 
Kah et al. 2012; Carenza et al. 2014b). Moreover, this aggregation and sedimentation in biological conditions could possibly affect the in vitro and in vivo toxicity assessments of NPs, and introduce problems of irreproducibility, difficulty interpreting the toxicity results, inaccurate determination of the dose for potential treatments and the reduced diagnostic efficiency (Petri-Fink et al. 2008; Butoescu et al. 2009). For these reasons, improving the stability of NPs in biologically relevant environments and establishing generalized protocols are of great importance and a major challenge.

In the last decades, many efforts were made to ensure stable NPs dispersions for in vitro or in vivo applications. A common used strategy to stabilize them is the functionalization of their surface using polymers or surfactants. Polyethylenimine (PEI), for example, as reported in many researches, is a well-known polymer that stabilizes efficiently NPs (Corti et al. 2008a; Hoskins et al. 2012), as well as polyethylene glycol (PEG), chitosan, dextran, citrate are other examples. (Kim et al. 2001; Janes et al. 2001; Liu et al. 2011; Safi et al. 2011). The choice of surfactant undoubtedly needs to be carefully evaluated to avoid any potential toxicity to cells and interference in the interpretation of NPs' cytotoxicity. (Ji et al. 2010; Kircheis et al. 2001). Moreover, in many cases, functionalization of NPs is not sufficient for their biological application purpose; the resulting NPs are stable in biological relevant media only for few hours. Wiogo et al. (Wiogo et al. 2012) showed that although the surface of SPIONs were modified by polymethacrylic acid, polyethylenimine, and branched oligoethylenimine, they aggregated and precipitated in biological media, despite the highly positively or highly negatively charged surface of the SPIONs.

Bovine serum albumin (BSA) is the most abundant protein in serum and one of the most important components in biological culture media. It has been reported as an efficient stabilizing agent to keep the dispersing state of several types of NPs in biological media, including gold NPs (Brewer et al. 2005; Dominguez-Medina et al. 2013), carbon nanotube (Porter et al. 2008), and titanium dioxide (Ji et al. 2010). Recently, a pH adjustment protocol was proposed by Guiot (Guiot and Spalla 2012) to stabilize $\mathrm{TiO}_{2}-\mathrm{NPs}$ with different surface coatings in biological media, where the $\mathrm{pH}$ of $\mathrm{TiO}_{2}-\mathrm{NPs}$ was first adjusted before adsorption of bovine serum albumin. As a result, the protocol showed robust ability in stabilizing $\mathrm{TiO}_{2}-\mathrm{NPs}$ in $\mathrm{LB}$ media (Lysogeny broth media, which is primarily used for the growth of bacteria). Meanwhile, other works demonstrated that addition of excess trisodium citrate $\left(\mathrm{Na}_{3} \mathrm{Cit}\right)$, one of the widely used additive in food and drug industry, into biological media could also stabilize SPIONs dispersions (Euliss et al. 2003; Luciani et al. 2009). These findings provide novel ways of further enhancing the dispersion stability of SPIONs in complex biological media. 
In the present work, water-dispersable tetramethylammonium hydroxide (TMAOH) coated SPIONs (TSPIONs) and citrate coated SPIONs (C-SPIONs) were synthesized in a facile, rapid, cost-effective and energetically favorable manner using microwave assisted method (MW). To the best of our knowledge, CSPIONs were the first time synthesized by this method. The obtained T-SPIONs and C-SPIONs were stabilized by adding BSA on their surface, which significantly enhanced their stability, with no aggregation phenomenon detected in $24 \mathrm{~h}$. Such stabilization protocol offers a fast and facile general methodology to obtain stable SPIONs in different biological media.

\section{Materials and Methods}

\section{Materials}

Iron (III) acetylacetonate $\left(\mathrm{Fe}(\mathrm{acac})_{3}, \geq 97.0 \%\right.$ ), tetramethylammonium hydroxide (TMAOH, 25 wt. \% in $\mathrm{H}_{2} \mathrm{O}$ ), trisodium citrate dihydrate $\left(\mathrm{Na}_{3} \mathrm{Cit}\right)$, bovine serum albumin (BSA, $\geq 98 \%$ ) and phosphate buffered saline (PBS, $1 \times$ ) were purchased from Sigma-Aldrich. Roswell Park Memorial Institute medium (RPMI), Dulbecco's Modified Eagle's Medium (DMEM), Fetal Bovine Serum (FBS) were obtained from Invitrogen. Benzyl alcohol was obtained from Scharlau. Acetone was bought from Panreac. If not stated otherwise, all materials were used as received.

\section{Microwave (MW) assisted synthesis of SPIONs}

MW-assisted method was used to synthesize pristine SPIONs in a CEM Discover reactor (Explorer 12-Hybrid) at a frequency of $2.45 \mathrm{GHz}$ and $300 \mathrm{~W}$. The synthesis process is slightly modified from Pascu et al. (Pascu et al. 2012). Briefly, $0.35 \mathrm{mmol} \mathrm{Fe}(\mathrm{acac})_{3}$ were dissolved completely in $4.5 \mathrm{~mL}$ anhydrous benzyl alcohol in a special MW-tube and vortexed for 30 seconds. Reaction tubes were transferred to the microwave reactor, the heating ramp was $5 \mathrm{~min}$ at $60^{\circ} \mathrm{C}$ and $10 \mathrm{~min}$ reaction at $180^{\circ} \mathrm{C}$, and further cooled down to $50^{\circ} \mathrm{C}$ in 3 min using compressed nitrogen.

\section{Synthesis of T-SPIONs}

$20 \mu \mathrm{L} 25$ wt. \% TMAOH was added to each MW-tube and sonicated for $1 \mathrm{~min}$. Then the solution of SPIONs is divided into 2 equal parts in $50 \mathrm{ml}$ centrifugal tubes. Each tube was filled with acetone and centrifuged at 6000 $\mathrm{rpm}$ for $30 \mathrm{~min}$. After removing the supernatant, $20 \mu \mathrm{L} 25 \mathrm{wt}$ \% TMAOH was added to each tube and sonicated for $1 \mathrm{~min}$, SPIONs were then washed and centrifuged three times. Collected SPIONs pellets were dried completely in a $60^{\circ} \mathrm{C}$ oven overnight, and redispersed in $2 \mathrm{ml}$ MQ $\mathrm{H}_{2} \mathrm{O}$ containing $10 \mu \mathrm{L} 25$ wt. \% TMAOH; $\mathrm{pH}$ of the obtained T- SPIONs colloidal dispersion was about 11. 


\section{Synthesis of C-SPIONs}

$150 \mu \mathrm{L} 10$ wt.\% $\mathrm{Na}_{3}$ Cit was added to each reaction MW-tube and sonicated for $1 \mathrm{~min}$. Then, the solution of SPIONs is divided into 2 equal parts in separate $50 \mathrm{ml}$ centrifugal tubes. Each centrifugal tube was filled up to $50 \mathrm{ml}$ with acetone to wash the SPIONs and the tubes were then subjected to centrifugation at $6000 \mathrm{rpm}$ for 30 min. After removing the supernatant, $150 \mu \mathrm{L} 10 \mathrm{wt} . \% \mathrm{Na}_{3} \mathrm{Cit}$ was added to each centrifugal tube and sonicated for $1 \mathrm{~min}$. SPIONs were washed and centrifuged again under the same conditions. Collected SPIONs pellets were dried completely in a $60^{\circ} \mathrm{C}$ oven overnight, and redispersed in $2 \mathrm{ml} \mathrm{MQ} \mathrm{H}_{2} \mathrm{O}$; pH of the obtained CSPIONs colloidal dispersion was about 8.3.

\section{Modified pH adjusted and BSA adsorption protocol}

A modified pH adjusted and BSA adsorption protocol based on Guiot et al was used (Guiot and Spalla 2012). Briefly, synthesized C-SPIONs and T-SPIONs were diluted to $2 \mathrm{mg} / \mathrm{mL}$ SPIONs dispersions in MQ $\mathrm{H}_{2} \mathrm{O}$. BSA solutions with a concentration of $0.5 \mathrm{w} / \mathrm{v} \%, 1 \mathrm{w} / \mathrm{v} \%, 2 \mathrm{w} / \mathrm{v} \%, 5 \mathrm{w} / \mathrm{v} \%, 10 \mathrm{w} / \mathrm{v} \%$ and $15 \mathrm{w} / \mathrm{v} \%$ were also prepared in MQ $\mathrm{H}_{2} \mathrm{O}$. For C-SPIONs, the $\mathrm{pH}$ of C-SPIONs was first adjusted to 11 by adding $0.01 \mathrm{M} \mathrm{NaOH}$, and then equal volumes of $2 \mathrm{mg} / \mathrm{mL}$ C-SPIONs dispersion and BSA solution were mixed rapidly and stirred on a vortex for $10 \mathrm{~min}$. Finally, $\mathrm{pH}$ of the mixture was adjusted to 7.4 (physiological pH value) by adding 0.05 mM $\mathrm{HNO}_{3}$ solution. As a result, $1 \mathrm{mg} / \mathrm{mL}$ BSA-C-SPIONs dispersion was obtained. The stability of the SPIONs dispersions after BSA addition was evaluated by dynamic light scattering (DLS) and Cryo transmission electron microscopy (Cryo-TEM).

For T-SPIONs, since the $\mathrm{pH}$ of T-SPIONs was already 11 , we directly mixed equal volume of $2 \mathrm{mg} / \mathrm{mL} \mathrm{T}$ SPIONs dispersion and BSA solution and incubated for $10 \mathrm{~min}$, then $\mathrm{pH}$ of the mixture was adjusted to 7.4. As a result, $1 \mathrm{mg} / \mathrm{mL}$ BSA-T-SPIONs dispersion was obtained.

\section{Stability of the obtained BSA-T-SPIONs and BSA-C-SPIONs dispersions in biological media}

$50 \mu \mathrm{L}$ of $1 \mathrm{mg} / \mathrm{mL}$ BSA-T-SPIONs or BSA-C-SPIONs were added to $0.95 \mathrm{~mL}$ biological media, mixed and vortexed, then diluted BSA-C-SPIONs or BSA-T-SPIONs dispersions in biological media at a SPIONs concentration at $50 \mu \mathrm{g} / \mathrm{ml}$ were obtained. Dynamic light scattering was used to monitor the stability of the prepared BSA-T-SPIONs and BSA-C-SPIONs dispersions in biological media during the period of $24 \mathrm{~h}$. It is noteworthy that the tested period of stability of BSA-T-SPIONs and BSA-C-SPIONs, as well as T-SPIONs and C-SPIONs in biological media was set at $24 \mathrm{~h}$, because in vitro toxicity assessment of NPs, in general, reports an incubation time of NPs with cells up to 24h (Soenen et al. 2011). 


\section{Characterization}

\section{DLS measurements}

DLS measurements were performed to monitor the changes of SPIONs in the hydrodynamic diameter (Dh), polydispersity Index (PDI) and diffusion coefficient, which are all relative to the stability of SPIONs dispersions over time.

Dh of the C-SPIONs and T-SPIONs were determined by using a Zetasizer Nano ZS (Malvern) device with a $\mathrm{He} / \mathrm{Ne} 633 \mathrm{~nm}$ laser at $25^{\circ} \mathrm{C}$ and at a SPIONs concentration of $50 \mu \mathrm{g} / \mathrm{mL}$. For each sample, 3 independent measurements were performed with 15 scans for each measurement.

\section{Zeta potential measurements}

Zeta potential was carried out to determine the changes of SPIONs in electrophoretic mobility (EPM) and zeta potential $(\zeta)$ on a Zetasizer Nano ZS (Malvern) apparatus with a zeta potential analyzer software. SPIONs solutions were placed in a disposable plastic cuvette, followed by collecting experimental data automatically. For each measurement, 20 cycles were collected, and at least 3 independent measurements were performed for each sample.

\section{Attenuated total reflectance Fourier transformed infrared spectra measurements (ATR-FTIR)}

ATR-FTIR was performed on a Spectrum RX1 FTIR Spectrometer (Perkin Elmer, USA), in the frequency range $4000-400 \mathrm{~cm}^{-1}$ with a resolution of $4 \mathrm{~cm}^{-1}$. All SPIONs samples were isolated from the solution by centrifugation, dried completely in the oven and placed on the crystal surface of the ATR-FTIR machine for measurements.

\section{TEM}

Size distribution, morphology and diffraction patterns of C-SPIONs and T-SPIONS were collected in JEOL JEM-1210 electron microscope at an operating voltage of $120 \mathrm{KV}$. TEM samples were prepared by placing one drop of the corresponding SPIONs dispersion on the copper grid for few seconds, blotting the copper grid with a filter paper and evaporate it completely at room temperature. Typically, about 200 to 300 different SPIONs were counted to depict the size distribution and the mean size of SPIONs.

\section{Cryo-TEM}

Samples for Cryo-TEM were prepared according to the method described by Hondow. (Hondow et al. 2012). Briefly, $3 \mu \mathrm{L}$ of $50 \mu \mathrm{g} / \mathrm{mL}$ BSA-C-SPIONs (diluted 20 times in MQ $\mathrm{H}_{2} \mathrm{O}$ ) was placed on a glow dischargetreated carbon support film (R1.2/1.3 Quantifoil Micro Tools GmBH), blotted and plunge freezed in liquid nitrogen, then samples were subsequently transferred to a Gatan 626 cryo-holder stored in liquid nitrogen. 
Cryo-TEM measurement was performed on a JEOL 2011 electron microscope at a temperature of about $-170{ }^{\circ} \mathrm{C}$ and $200 \mathrm{kv}$ acceleration voltage.

\section{Negative staining TEM}

Adsorption of BSA on T-SPIONs and C-SPIONs were visualized by performing negative staining TEM. (Ye et al. 2006) Briefly, BSA-T-SPIONs or BSA-C-SPIONs were purified from the excess BSA solution by centrifugation at 10000 ref for $3 \mathrm{~h}$ and redispersed in $\mathrm{MQ} \mathrm{H}_{2} \mathrm{O}$. (Casals et al. 2010) Then one drop of the purified BSA-C-SPIONs was placed on a carbon-caoted grid and then drained off with filter paper. Subsequently, $5 \mu \mathrm{L}$ of $2 \%$ uranyl acetate was placed on the grid for $1 \mathrm{~min}$ before drained off. The grid was then placed in a 2011 JEOL electron microscope.

\section{Results and discussion}

\section{Synthesis and characterization of C-SPIONs and T-SPIONs}

In the present work, C-SPIONs were synthesized for the first time by a facile, rapid, cost effective and energetically favorable microwave assisted method, modifying the previously reported for T-SPIONs (Pascu et al. 2012). In this method, bare SPIONs were first synthesized in benzyl alcohol by a single step microwave heating method, followed by coating the SPIONs surface with citrate and TMAOH ligands, respectively.

Fig.1a show representative TEM images of the as-synthesized spherical T-SPIONs and C-SPIONs. Particle size histograms of T-SPIONs and C-SPIONs (Fig. 1b) indicate the high monodispersity of both SPIONs, with an identical SPIONs core of $6 \pm 1 \mathrm{~nm}$. Selected area electron diffraction (Fig. 1c) reveal the crystallinity of both SPIONs. The electron diffraction rings correspond to (220), (311), (400), (422), (511) and (440) planes, and they indicate the inverse spinel structure (magnetite, maghemite) of C-SPIONs and T-SPIONs. DLS intensityweighted size distributions further evidenced the monodispersity of SPIONs (Fig. 1d), with Dh of about $26 \pm 1$ $\mathrm{nm}$ for T-SPIONs and $14 \pm 1 \mathrm{~nm}$ for C-SPIONs. Zeta potential values for T-SPIONS and C-SPIONS are -54 $\mathrm{mV}$ and $-36 \mathrm{mV}$, respectively (see Supporting Information Table S1 and Table S2), indicating both T-SPIONs and C-SPIONs are charged negative. 


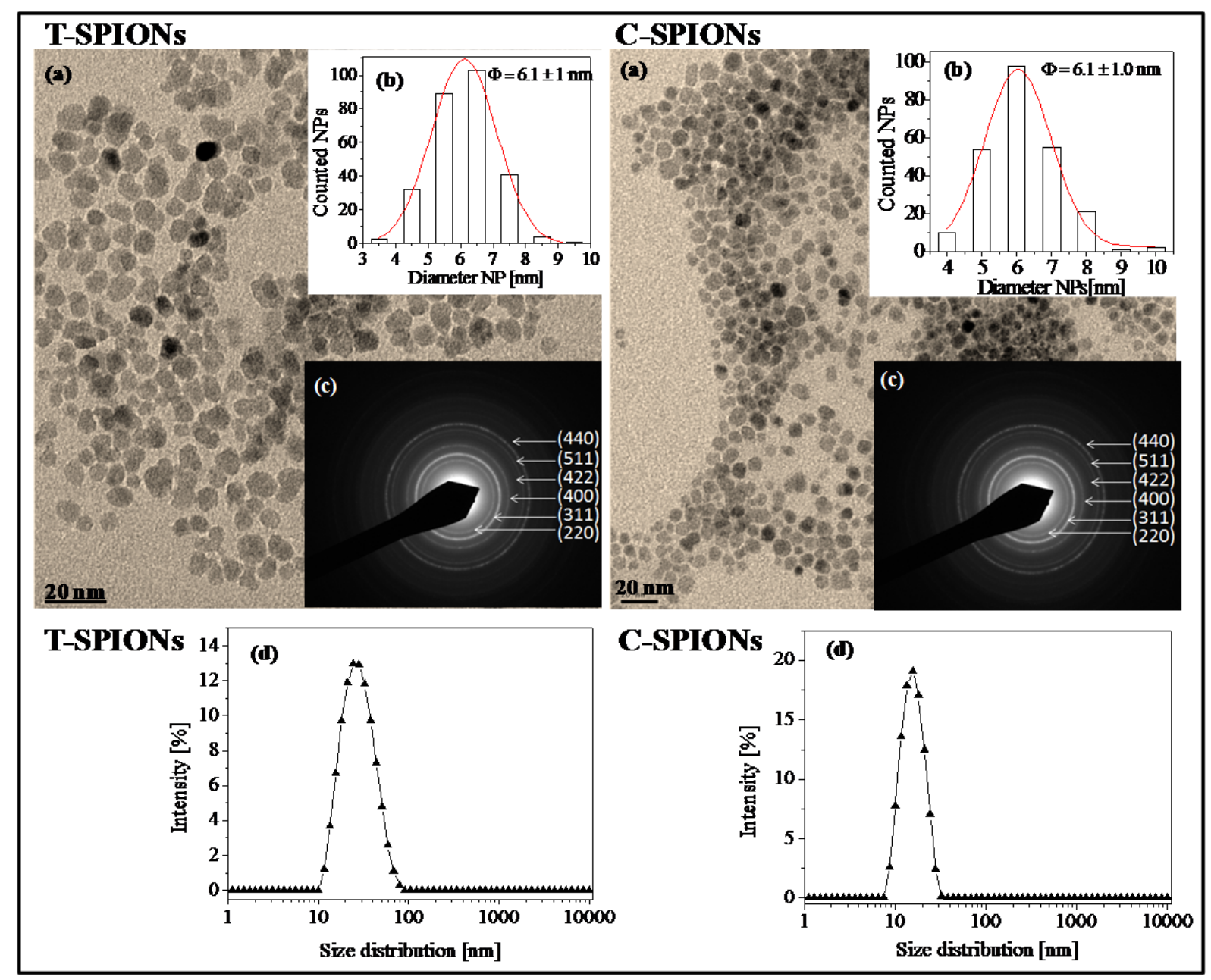

Fig. 1 (a) TEM images, (b) particle size histogram, (c) electron diffraction and (d) DLS intensity-weighted size distribution of T-SPIONs and C-SPIONs.

FTIR spectrum of T-SPIONs and C-SPIONs present adsorption bands at $620 \mathrm{~cm}^{-1}$ and $635 \mathrm{~cm}^{-1}$, respectively (Fig. 2A-c and 2B-c), these bands are attributed to the Fe-O stretching vibration, evidencing the existence of magnetite (Namduri and Nasrazadani 2008). Typical band for TMAOH at $944 \mathrm{~cm}^{-1}$ (Fig 2A-a), characteristic of the asymmetric methyl deformation mode of C-N on TMAOH (Ouasri et al. 2002), was found to shift to 975 $\mathrm{cm}^{-1}$ (Fig. 2A-c), indicating the existence of TMAOH on T-SPIONs. Peaks at $1570 \mathrm{~cm}^{-1}$ and $1450 \mathrm{~cm}^{-1}$ (Fig. 2B-a) are characteristic of the $\mathrm{RCO}_{2}$ symmetric and asymmetric stretches (Sasidharan et al. 2013), though both peaks show a slight shift from $1579 \mathrm{~cm}^{-1}$ and $1415 \mathrm{~cm}^{-1}$ (Fig 2B-c), respectively, suggesting citrate ligand is present on the SPIONs surface. 

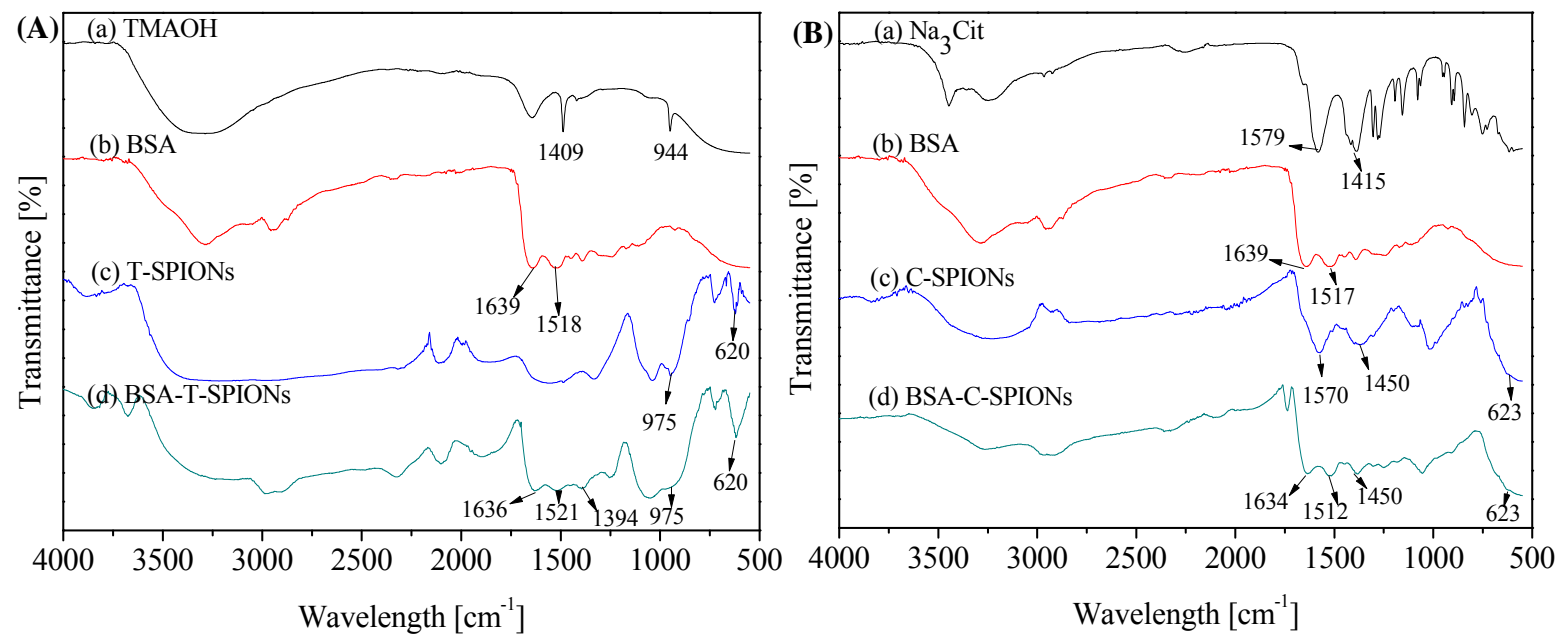

Fig. 2 (A) FTIR spectrum of (a) TMAOH, (b) BSA, (c) T-SPIONs and (d) BSA-T-SPIONs; (B) FTIR spectrum of (a) $\mathrm{Na}_{3} \mathrm{Cit}$, (b) BSA, (c) C-SPIONs and (d) BSA-C-SPIONs.

\section{Stability of T-SPIONs and C-SPIONs in biological media}

In the present investigation, stability of T-SPIONs and C-SPIONs were assessed in biologically relevant media, PBS, RPMI and DMEM, at a SPIONs concentration of $50 \mu \mathrm{g} / \mathrm{mL}$. We chose PBS, RPMI and DMEM since they are media extensively used in in vitro toxicity and stability assays of SPIONs and differ significantly in their ionic strength and salt content like $\mathrm{Ca}^{2+}$ and $\mathrm{Mg}^{2+}$. The salt content in terms of $\mathrm{Ca}^{2+}$ concentration, for example, follows the order of PBS $<$ RPMI $<$ DMEM, with a value of $0 \mathrm{mM}, 0.4 \mathrm{mM}$ and $1.8 \mathrm{mM}$, respectively (Lee et al. 2002).

Fig. 3 shows the Dh evolution of T-SPIONs and C-SPIONs in $\mathrm{H}_{2} \mathrm{O}$ and different biological media as a function of time. Both SPIONs show good stability in $\mathrm{H}_{2} \mathrm{O}$ since Dh doesn't change up to 3 months (data not shown), this is attributed to the highly negative charged surface of both SPIONs. When incubated in biologically relevant media, however, T-SPIONs destabilized and precipitated rapidly (Fig. 3a). In all the cases, a similar behavior is observed. Dh of T-SPIONs in RPMI, for instance, goes up sharply from $25 \mathrm{~nm}$ to about $2800 \mathrm{~nm}$ in $2 \mathrm{~min}$, and subsequently T-SPIONs precipitated. Moreover, the addition of 10\% FBS to RPMI and DMEM media could not prevent the sedimentation of T-SPIONs, as Dh of T-SPIONs in RPMI-FBS and DMEM-FBS shows an increase within $10 \mathrm{~min}$. Consistent with the dramatic increase in Dh, significant decrease in diffusion coefficient, obvious increase in zeta potential $(\zeta)$ and electrophoretic mobility (EPM) were also detected (Supporting Information Table S1), confirming the destabilization of T-SPIONs in these media. On the other hand, C-SPIONs displayed a different aggregation behavior in these biological media. C-SPIONs show excellent stability in PBS and RPMI, as Dh remains unchanged up to $24 \mathrm{~h}$ (Fig. 3b). C-SPIONS in 
DMEM, unfortunately, just show a transient stability. Dh unchanged in the first initial $1 \mathrm{~h}$, although increased rapidly to about $250 \mathrm{~nm}$ in the following $1 \mathrm{~h}$, and reached up to $2800 \mathrm{~nm}$ in $4 \mathrm{~h}$. In RPMI-FBS and DMEM-FBS, Dh of C-SPIONs show a slight increase in the initial $30 \mathrm{~min}$ and remains flat in the later time, also suggesting the good stability of C-SPIONs in the serum containing media, the slight increase in both cases could be interpreted as the adsorption of serum proteins on the surface of C-SPIONs. Changes in diffusion coefficient, zeta potential $(\zeta)$ and EPM (Supporting Information Table S2) further confirm these findings.
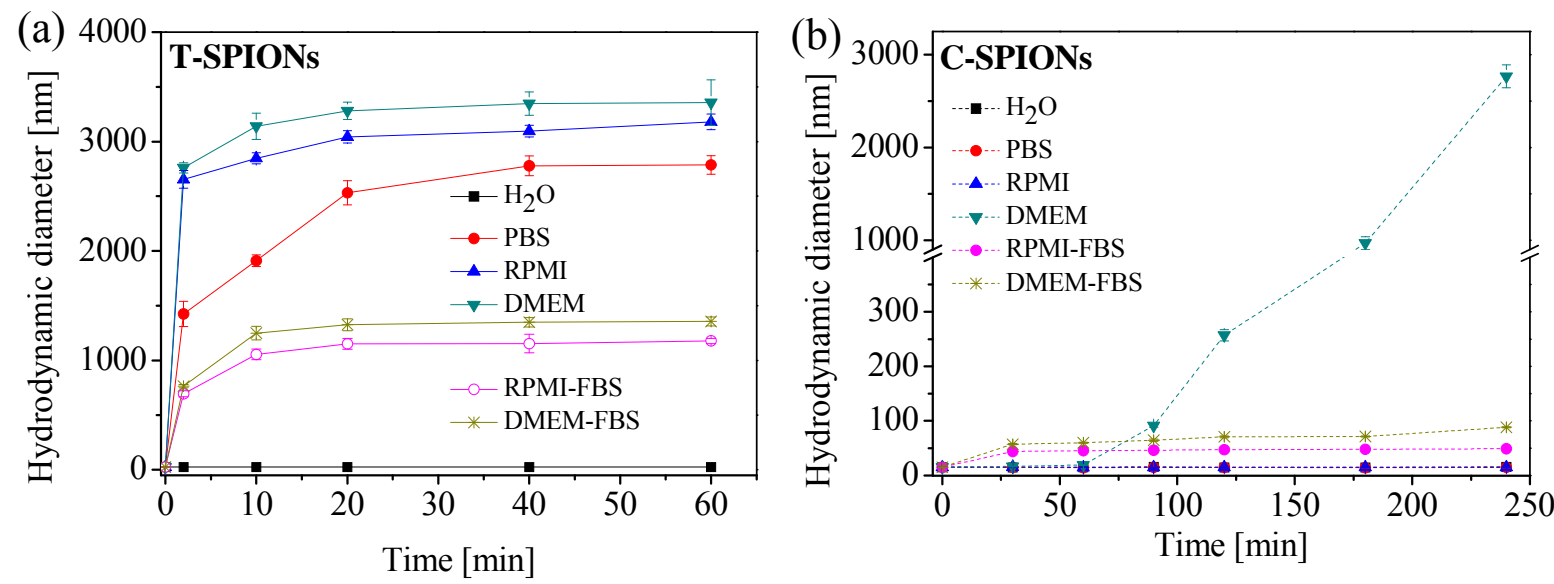

Fig. 3 Stability of (a) T-SPIONs and (b) C-SPIONs in $\mathrm{H}_{2} \mathrm{O}$, PBS, RPMI-FBS and DMEM-FBS in terms of Dh as a function of time.

From the above results, we can see clearly that C-SPIONs show better stability than T-SPIONs in biological media, then it could be concluded that surface coating greatly influences the stability of SPIONs in these media. Although both T-SPIONs and C-SPIONs show highly negative charged surface, the adsorption mechanism of TMAOH and citrate ligands on SPIONs surface are quite different. For T-SPIONs, TMAOH cations adsorb onto SPIONs surface through electrostatic interaction and this results in an electrostatic double layer which provide stability for SPIONs in aqueous solution (Euliss et al. 2003). However, this electrostatic double layer can be easily disassembled by the species in biological media like salts and biomolecules. As for C-SPIONs, the three carboxylate groups of citrate ligand have strong coordination affinity to Fe(III) ions, some of the carboxylate groups strongly coordinate to SPIONs surface, the rest carboxylate groups extend into the water, providing a more robust coating and higher stability to SPIONs in $\mathrm{H}_{2} \mathrm{O}$ and even in some complex media like PBS and RPMI (Nigam et al. 2011).

The stability and aggregation behavior of SPIONs in biological media was reported to be not only dependent on the SPIONs surface coating, but also on the nature of the surrounding media (Petri-Fink et al. 2008; 
Eberbeck et al. 2010). Some research reported that NPs aggregate and sediment immediately when exposed to biological media (Wells et al. 2012; Allouni et al. 2009; Geppert et al. 2012), where high ionic strength was considered an important factor to cause the NPs aggregation. When NPs are in contact with these media, surface charges on their surface were suppressed by the high ionic strength of salts presented in these media, decreasing the electrostatic repulsion significantly and subsequently precipitating the NPs (Casals et al. 2010; Wiogo et al. 2012). In particular, Safi M. and coworkers found that the divalent calcium and magnesium cations presenting in the cell media significantly reduce the stability of the C-SPIONs through the complex reaction with the citrate ligands (Safi et al. 2011). Similar significant aggregation phenomenon happened to our synthesized T-SPIONs in biological media and C-SPIONs in DMEM, despite the highly negatively charged surface of both T-SPIONs and C-SPIONs. This suggests that the surface coating of C-SPIONs and T-SPIONs are not enough to maintain their stability when exposed to biologically relevant media with high salts content, which could in turn limit their biological applications. Thus, improvement of the stability of SPIONs in biologically relevant media is of significant importance.

\section{Stabilization of T-SPIONs and C-SPIONs in biological media with a pH adjusted and BSA adsorption protocol}

To improve further the stability of T-SPIONs in biological media and C-SPIONs in DMEM, we adsorbed BSA to the SPIONs. The adsorption of BSA on NPs surface could offer higher stability and biocompatibility rather than using strategies as poly (methyl methacrylate) (PMMA), PEI or other organic layers (Khullar et al. 2012; Dominguez-Medina et al. 2013). In particular, a modified pH adjusted and BSA adsorption protocol based on that proposed by Guiot (Guiot and Spalla 2012) was used in this work to modify the T-SPIONs and C-SPIONs.

The protocol presented by Guiot et al (Guiot and Spalla 2012) was in fact based on the colloidal stability theory, in particular the DLVO theory, which indicates electrostatic repulsion force, rather than Van der Waals attraction force is responsible for the colloidal stability of NPs dispersions. In other words, once the electrostatic repulsion increased, the stability of the NPs dispersions improved. Following this principle, we first adjusted the $\mathrm{pH}$ of SPIONs at 11 where SPIONs and BSA exhibit a net charge of identical sign, and then we added BSA to the SPIONs dispersion. BSA with a negative net charge at pH 11 can still be attached to the negative charge NPs due to the intrinsic amphiphilic character of the protein as reported previously. (Brewer et al. 2005; Wiogo et al. 2012) The addition of enough BSA in the SPIONs solutions, allows the coverage of the SPIONs while maintaining its stability. The BSA coverage avoids the aggregation of the SPIONs solutions once we change the solutions to physiological pH. (Dominguez-Medina et al. 2013) 
The $\mathrm{pH}$ of the synthesized C-SPIONs was first adjusted to 11 by adding $0.1 \mathrm{M} \mathrm{NaOH}$ ( $\mathrm{pH}$ of synthesized TSPIONs was already 11).At pH 11, the zeta potential measurement of BSA and C-SPIONs decreased from - 22 $\mathrm{mV}(\mathrm{pH} 7.4)$ to $-28 \mathrm{mV}$ and $-36 \mathrm{mV}(\mathrm{pH} 8.3)$ to $-42 \mathrm{mV}$ respectively. Then, SPIONs dispersions were incubated with BSA solution for $10 \mathrm{~min}$, and then $\mathrm{pH}$ of the mixture was adjusted to the physiological value of 7.4. BSA-T-SPIONs and BSA-C-SPIONs dispersions prepared are extremely stable due to the electrostatic repulsion among them and the BSA coverage on the SPIONs surface.

$50 \mu \mathrm{L}$ of the obtained BSA-T-SPIONs and BSA-C-SPIONs $(1 \mathrm{mg} / \mathrm{mL})$ were diluted 20 times in water to a final concentration of $50 \mu \mathrm{g} / \mathrm{mL}$ and their stability was monitored by DLS over $24 \mathrm{~h}$. DLS intensity-weighted size distributions (Supporting Information Fig. S1a and Fig. 4a), Dh, diffusion coefficient and diffusion time remain unchanged over 24h (Supporting Information Table S3 and Table S4), implying high stability of the prepared BSA-T-SPIONs and BSA-C-SPIONs dispersions over time. In the DLS measurement there is no peak observed at $8 \mathrm{~nm}$, from the excess of BSA, confirming that the 20 times dilution decreases the BSA content in the samples. Different literature suggest the existence of two layers contributing to the stabilization of NPs with BSA: the hard protein corona, strongly interacting with the NPs'surface and the soft protein corona, BSA molecules loosely bounded to the BSA-NPs. (Casals et al. 2010). Therefore the dilution of our samples and the excess of the BSA could contribute to the stabilization of the NPs. Cryo-TEM is a potential technology that provides a representative view of the real dispersing state of NPs in solution, therefore, Cryo-TEM measurements were also performed to reveal the stability of the obtained BSA-C-SPIONs, as it is shown in Fig. 4b. BSA-C-SPIONs exist in the solution as small aggregates but well separated from each other, suggesting the well dispersing state of BSA-C-SPIONs in solution even after $24 \mathrm{~h}$ incubation, this might due to the electrostatic repulsion provided by their negative charged surfaces.

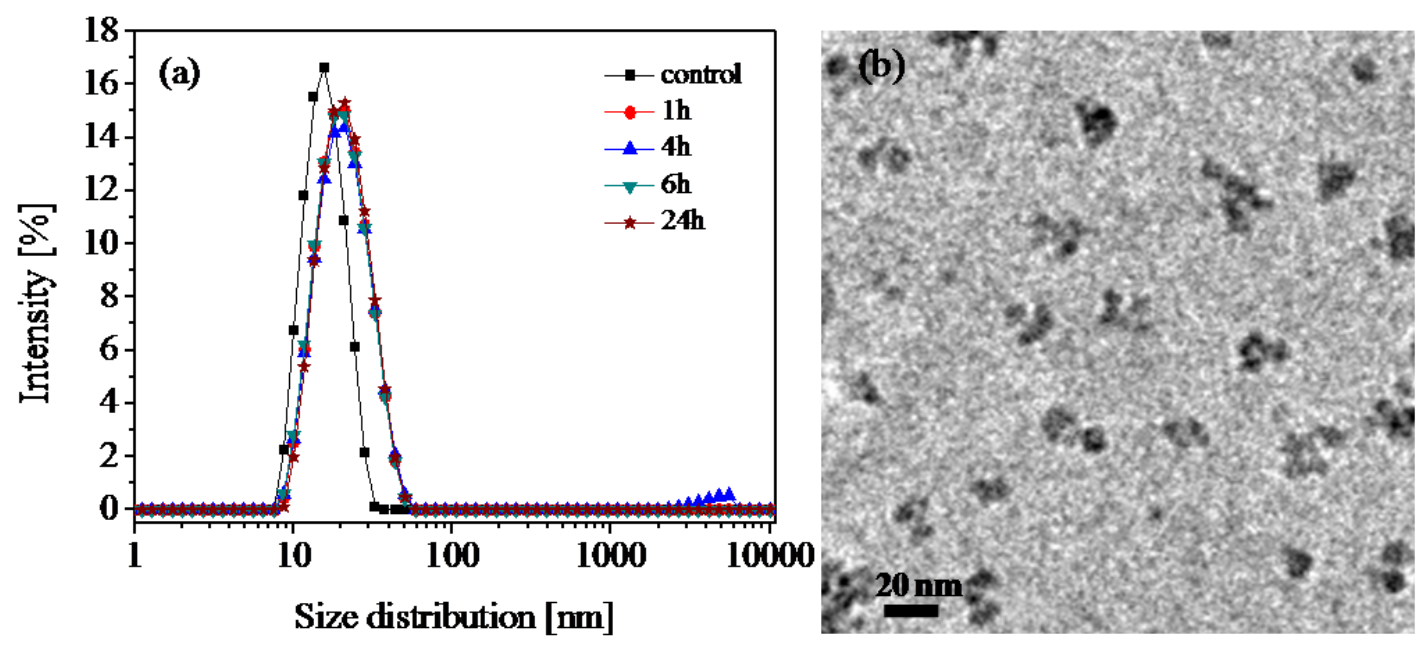

Fig. 4 (a) DLS intensity-weighted size distributions of C-SPIONs (control) and the prepared BSA-C-SPIONs 
at an evaluated time of $1 \mathrm{~h}, 4 \mathrm{~h}, 6 \mathrm{~h}$, and $24 \mathrm{~h}$ in water; (b) Cryo-TEM image of the prepared BSA-C-

SPIONs after $24 \mathrm{~h}$ preparation in water.

To test the practical effect of our protocol, we evaluated the stability of BSA-T-SPIONs and BSA-C-SPIONs in PBS, RPMI and DMEM. Firstly, they were diluted 20 times to a final BSA-SPIONs concentration at $50 \mu \mathrm{g} / \mathrm{mL}$ with the appropriate media and we monitored their aggregation evolution using DLS over 24h. BSAT-SPIONs and BSA-C-SPIONs are well dispersed and stable in PBS, without any increase in Dh observed over the tested period, as well as the diffusion coefficients and diffusion times (Fig. 5a, Supporting Information Fig. S1b, Fig. S2a, Table S5 and S6).

Stability of BSA-T-SPIONs and BSA-C-SPIONs $50 \mu \mathrm{g} / \mathrm{mL}$ solutions in RPMI and DMEM, which have higher salt content compared to PBS, was tested. Intensity-weighted size distributions of BSA-T-SPIONs in RPMI and DMEM show a gradual shift toward larger size as incubation time increased, Dh increases from 24 $\mathrm{nm}$ to $100 \mathrm{~nm}$ in RPMI over 24h and to $220 \mathrm{~nm}$ in RPMI (Supporting Information Fig. S1c and S1d). The increase in $\mathrm{Dh}$ is also confirmed by the decrease diffusion coefficient and the increased diffusion time (Supporting Information Table S7 and S8). Although BSA-T-SPIONs increased in size in RPMI and DMEM, the present size is still desirable for in vitro toxicity studies when compared to the rapid and significant aggregation behavior of the synthesized T-SPIONs in the same media. Moreover, no sedimentation appeared in 24h for BSA-T-SPIONs in both media (Supporting Information Fig. S2a).
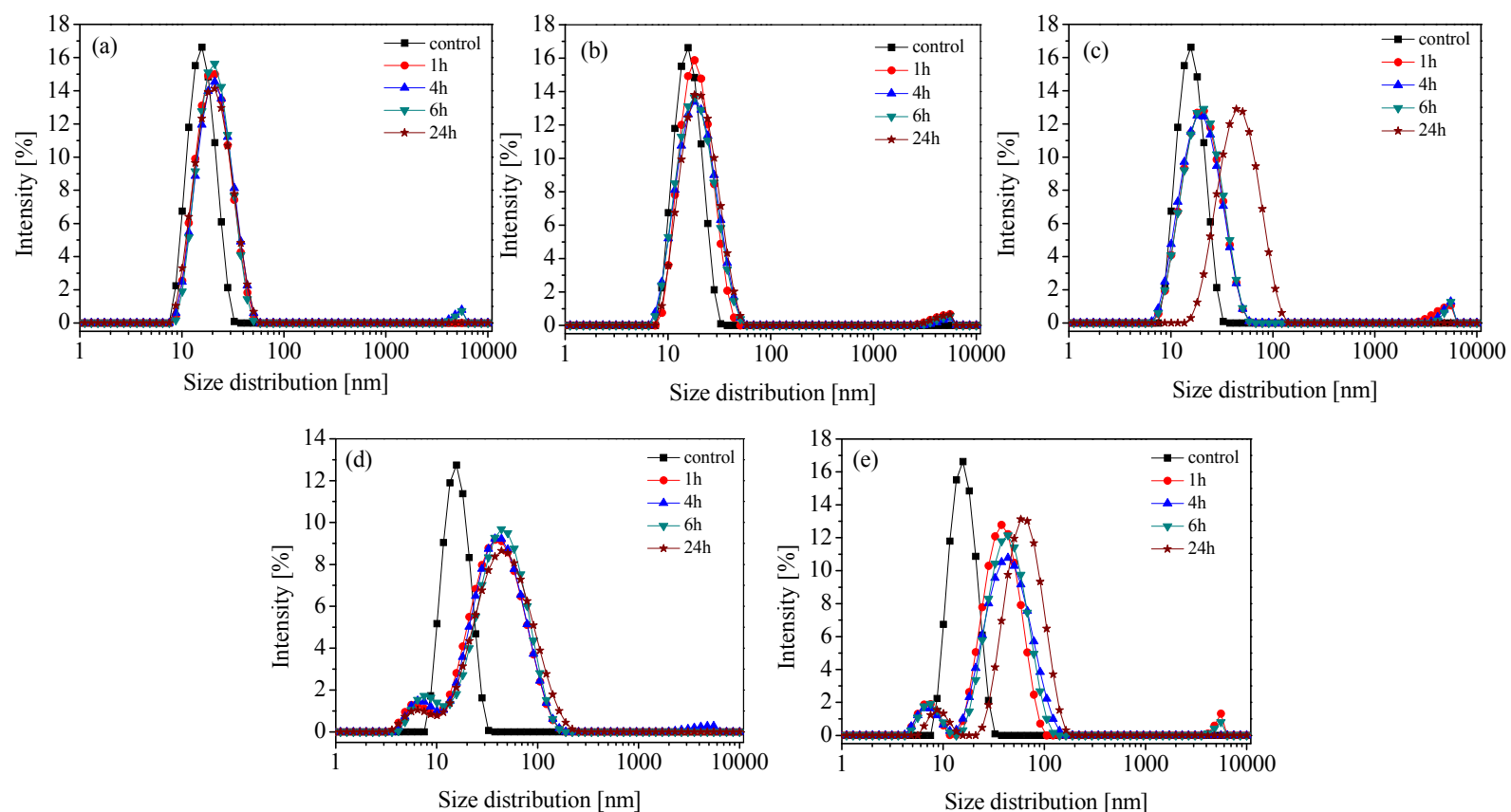
Fig. 5 DLS intensity-weighted size distributions of C-SPIONs (control) and BSA-C-SPIONs in (a) PBS; (b) RPMI; (c) DMEM; (d) RPMI-FBS and (e) DMEM-FBS at an evaluated time of 1h, 4h, 6h, and $24 \mathrm{~h}$.

As expected, BSA-C-SPIONs remain extremely stable in RPMI and DMEM even after 24h incubation. Intensity-weighted size distributions of BSA-C-SPIONs in RPMI as a function of time superimposed to each other (Fig. 5b), diffusion coefficient and diffusion time also kept unchanged (Supporting Information Table S9), indicating the identical distribution of the BSA-C-SPIONs in 24h. Dh of BSA-C-SPIONs in DMEM only increased few nanometers (Fig. 5c), whereas synthesized C-SPIONs show dramatic aggregation, implying the robustness of the $\mathrm{pH}$ adjusted and BSA adsorption protocol. Slight changes in diffusion coefficient and diffusion time are found for BSA-C-SPIONs in DMEM, confirming the excellent stability. (Supporting Information Table S10)

Media for cell culture are always supplemented with serum proteins, therefore we further tested the stability of BSA-T-SPIONs and BSA-C-SPIONs under serum conditions, RPMI-FBS and DMEM-FBS respectively. In all these cases, DLS intensity-weighted size distributions as a function of time exhibit minor shifts in Dh, as well as slight changes in diffusion coefficient and diffusion time, which clearly precludes the possibility of aggregation or sedimentation of BSA-T-SPIONs and BSA-C-SPIONs in these media (Fig. 5d and 5e, Supporting Information Fig. S1e, S1f, S2a, Table S11, S12, S13 and S14).

In the present investigation, we also found that to endow SPIONs dispersions with sufficient stability in the biological media, the necessary concentration of BSA in the protocol varied significantly (Table 1). For both SPIONs, the necessary concentration of BSA followed the order of in DMEM $>$ RPMI $>$ PBS $>$ RPMI-FBS and DMEM-FBS. We attribute this to the different compositions of these tested media. As mentioned before, the salt content in terms of $\mathrm{Ca}^{2+}$ concentration follows the order of PBS $<$ RPMI $<$ DMEM $\left(\mathrm{Mg}^{2+}\right.$ concentration also follows the same order) and it is the divalent calcium and magnesium cations in biological media that are reported to mainly cause the SPIONs aggregation though interacting with the surface coating on the SPIONs surface. BSA added to the SPIONs dispersions could complex with these divalent cations, protecting the SPIONs surface by screening these divalent cations from interacting directly with the SPIONs surface, thus keep the SPIONs stable in the tested media. Hence, in biological media with higher concentration of divalent cations, it is expected that the amount of BSA needed should be also higher. For this reason, the amount of BSA needed is the highest in DMEM, followed by RPMI and PBS. Furthermore, Ji et al. (Ji et al. 2010) found that compositions in FBS, like globulin and transferrin, exhibit a synergistic effect on stabilizing the NPs dispersions with BSA. Therefore, in RPMI-FBS and DMEM-FBS, FBS itself served as the additional stable 
reagent in addition to BSA, thus the necessary concentration of BSA is the least compared to those media in the absence of $10 \%$ FBS. The amount of FBS is clearly detected in the DLS intensity-weighted size distributions in Fig. 5d and Fig. 5e.

\begin{tabular}{cccccc} 
& \multicolumn{5}{c}{ Concentration of BSA needed $(\mathrm{w} / \mathrm{v} \%)$} \\
\cline { 2 - 6 } SPIONs & PBS & RPMI & DMEM & RPMI-FBS & DMEM-FBS \\
& 2 & 10 & 15 & 1 & 1 \\
T-SPIONs & 1 & 2 & 5 & 0.5 & 0.5 \\
\hline
\end{tabular}

Table 1 The necessary concentration of BSA needed for T-SPIONs and C-SPIONs in different media by using the $\mathrm{pH}$ adjusted and BSA adsorption protocol.

Moreover, we observed that in all the tested media, the necessary concentration of BSA needed for CSPIONs is always lower than that for T-SPIONs. We relate this to the different surface coating of C-SPIONs and T-SPIONs. As it was concluded before, citrate ligands exhibit stronger ability of resisting against high salt and high ionic strength than TMAOH. Previous studies also revealed that nonspecific binding of BSA to selfassembled monolayers following the order of hydrophobic $>\mathrm{COO}^{-}>\mathrm{NH}^{3+}>\mathrm{OH}^{-}>$ethylene glycol (Nakata et al. 1996; Moulin et al. 1999), which indicates BSA has a preference and a higher affinity for binding to SPIONs surface with citrate ligands (with three $\mathrm{COO}^{-}$groups per molecule) than those with TMAOH coating (with one $\mathrm{OH}^{-}$per molecule). As a result, more BSA is envisaged to bind more effectively on C-SPIONs than on TSPIONs under the same BSA concentration. For those reasons, using less BSA and we could get the same or even better stability of C-SPIONs in biological media than T-SPIONs.

\section{Stabilization of C-SPIONs and T-SPIONs in biological media by adding excess $\mathrm{Na}_{3} \mathrm{Cit}$}

As a comparison, addition of excess $\mathrm{Na}_{3} \mathrm{Cit}$ to enhance the stability of the T-SPIONs and C-SPIONs in biological media was also used, because some researches indicated its enhancing effects (Euliss et al. 2003; Luciani et al. 2009). Briefly, a solution of $50 \mu \mathrm{g} / \mathrm{mL}$ SPIONs in biological media and with a $10 \mathrm{mM} \mathrm{Na}_{3} \mathrm{Cit}$ were prepared and their stability was monitored by DLS over $24 \mathrm{~h}$. Results show that adding $10 \mathrm{mM}$ excess $\mathrm{Na}_{3} \mathrm{Cit}$ could not prevent the aggregation of T-SPIONs in all of the tested media, T-SPIONs destabilized and precipitated in $1 \mathrm{~h}$ (Supporting Information Fig S2b). While stability of C-SPIONs was maintained in $24 \mathrm{~h}$ by adding $10 \mathrm{mM}$ excess $\mathrm{Na}_{3}$ Cit in DMEM as well as in the other media (Fig. 6). Dh doesn't show any increase in PBS-Na ${ }_{3}$ Cit within $24 \mathrm{~h}$, only slight increase in RPMI-Na ${ }_{3}$ Cit and DMEM-Na ${ }_{3}$ Cit was found. In RPMI-FBS- 
$\mathrm{Na}_{3} \mathrm{Cit}$ and DMEM-FBS-Na $\mathrm{Cit}$, Dh increase to about $35 \mathrm{~nm}$ in the initial $1 \mathrm{~h}$, and kept unchanged in the following $24 \mathrm{~h}$. The increase in Dh during the beginning $1 \mathrm{~h}$ is due to the formation of protein corona on CSPIONs surface, demonstrated by Walczyk (Walczyk et al. 2010) who described that protein corona on NPs surface formed in a relatively stable manner over a period of one hour.

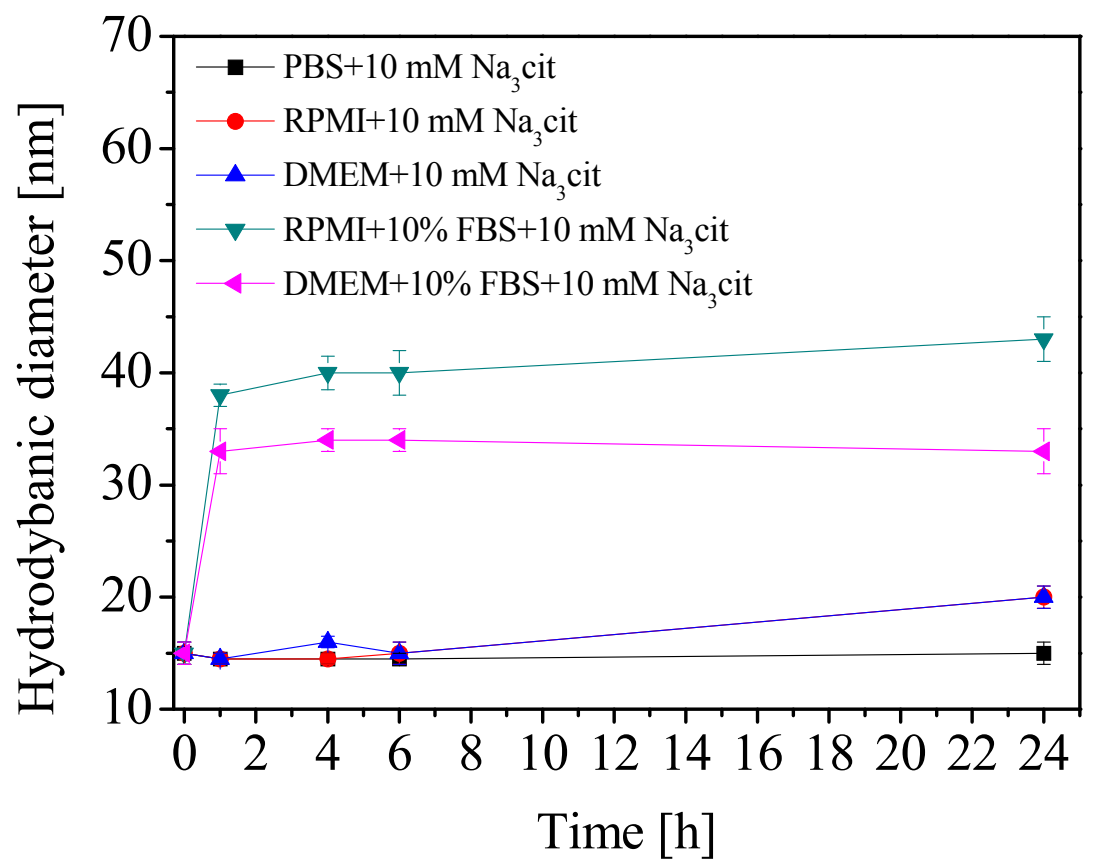

Fig. 6 Graph showing the changes in hydrodynamic size of C-SPIONs in PBS-Na ${ }_{3}$ Cit, RPMI-Na ${ }_{3}$ Cit, DMEM$\mathrm{Na}_{3} \mathrm{Cit}$, RPMI-FBS-Na $\mathrm{C}_{3} \mathrm{Cit}$ and DMEM-FBS-Na $\mathrm{Cit}_{3}$ at an evaluated time of $24 \mathrm{~h}$.

In summary, the modified $\mathrm{pH}$ adjusted and BSA adsorption protocol shows excellent prospect in preparing extremely stable SPIONs dispersions in several biologically relevant media, which are desirable for reliable in vitro and in vivo toxicity assessments. Although, the addition of excess $\mathrm{Na}_{3} \mathrm{Cit}$ could prevent C-SPIONs from aggregation in DMEM and other media, the stabilization effect is limited. Moreover, high concentration of $\mathrm{Na}_{3} \mathrm{Cit}$ in biological media is reported to produce risky effects to the growth of the cells (Freese et al. 2012; Uboldi et al. 2009). Therefore, the modified BSA adsorption and pH adjusted protocol seems a general and more suitable method to improve the stability of SPIONs in biological media.

\section{Characterization of BSA layer on C-SPIONs and T-SPIONs}

We further characterized the binding mechanism of the BSA layer on T-SPIONs and C-SPIONs surface and the interaction mechanism of SPIONs and BSA. Fig. 7a shows the changes in Dh of T-SPIONs and C-SPIONs before and after performing the $\mathrm{pH}$ adjusted BSA adsorption protocol. Diluted BSA-T-SPIONs shows $5 \mathrm{~nm}$ 
increase in $\mathrm{Dh}$ and $6 \mathrm{~nm}$ for diluted BSA-C-SPIONs. The dimension of BSA is reported to be $(8 \times 8 \times 8 \times 3.4$ $\mathrm{nm}$ ) as a triangular equilateral prism (He and Carter 1992), the increase of Dh for both SPIONs after using the protocol just corresponds to the shorter dimension of BSA, therefore, we conclude that a BSA monolayer was formed on both SPIONs surface in the form of triangular base. The result obtained here is in agreement with previous findings using BSA as a model protein and Au NPs as model NPs (Dominguez-Medina et al. 2013; Kohli et al. 2013). To provide clearer evidence of the formed BSA monolayer on SPIONs surface, we present negative staining TEM data for the purified BSA-T-SPIONs and BSA-C-SPIONs in water. Representative negative staining TEM images (Fig. 7c and 7d) show clear evidence of this BSA monolayer, which completely covers the both SPIONs surface. Moreover, after measuring the thickness of these images, an increase in diameter of $6 \pm 1 \mathrm{~nm}$ was computed, which is consistent with the data obtained by DLS, suggesting a BSA monolayer with thickness about 3nm was formed on T-SPIONs and C-SPIONs surface.
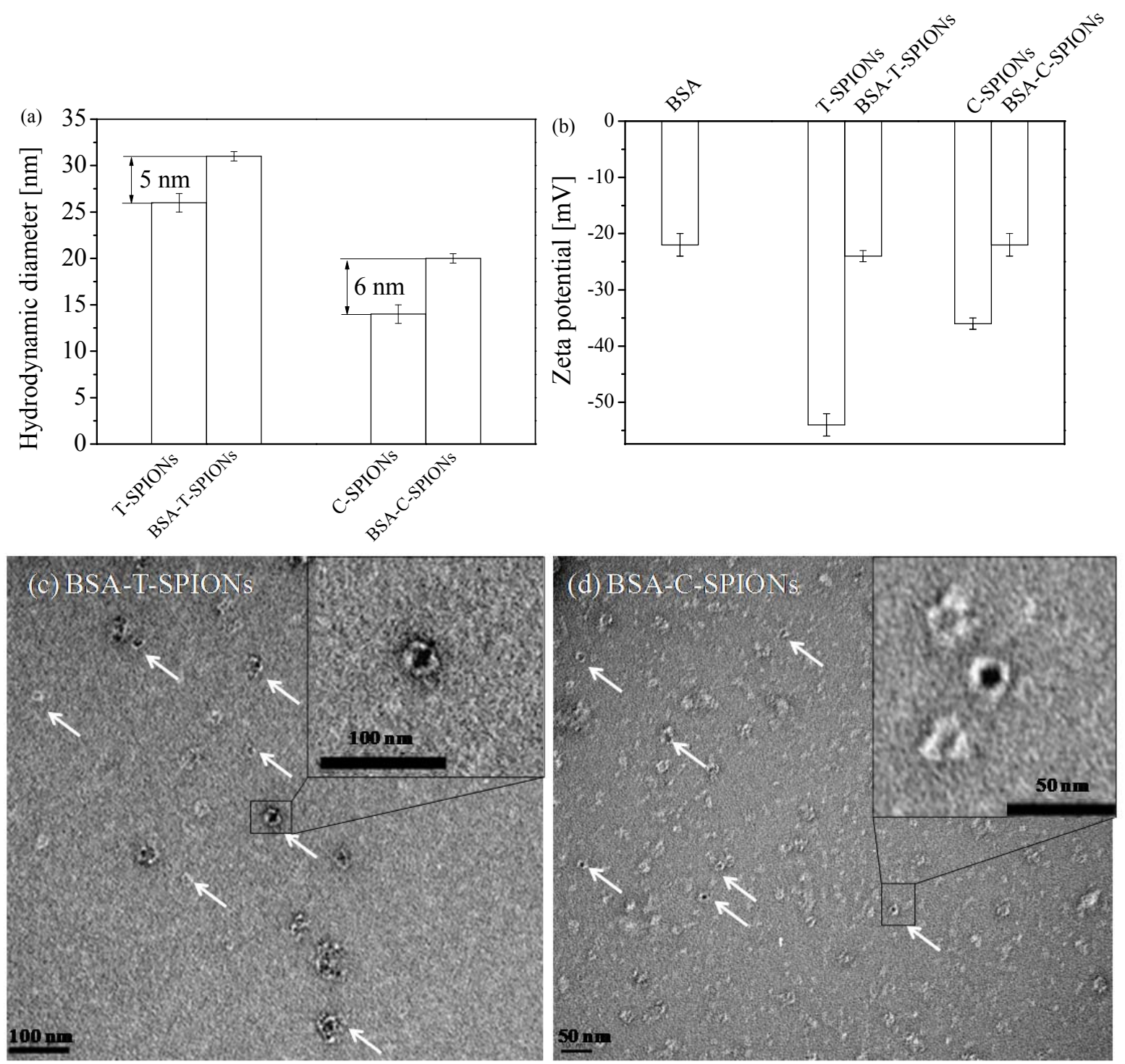
Fig. 7 (a) Hydrodynamic size and (b) zeta potential of T-SPIONs, BSA-T-SPIONs, C-SPIONs and BSA-CSPIONs. Negative staining TEM images of (c) BSA-T-SPIONs and (d) BSA-C-SPIONs.

The formation of protein corona on SPIONs surface will likely change their zeta potential and the isoelectric point (IEP) (Natte et al. 2013), thus the change in zeta potential of C-SPIONs and T-SPIONs before and after performing the protocol should also evidence the BSA adsorption on their surface. The zeta potential value of T-SPIONs and C-SPIONs (-54 $\mathrm{mV}$ and $-42 \mathrm{mV}$, respectively) increased to $-24 \mathrm{mV}$ and $-22 \mathrm{mV}$ for BSA-T SPIONs and BSA-C-SPIONs respectively (Fig. 7b).

Recent studies on the interaction of Au NPs and BSA revealed two possible mechanisms: 1) strong binding of BSA to Au NPs due to the electrostatic attraction between positive residues of BSA and negative charged surface coating on Au NPs surface (Sen et al. 2011) and 2) the surface coating on Au NPs is removed by BSA upon absorption, amino acids on BSA, in particular cysteine, bind directly to the Au NPs surface (Brewer et al. 2005). To the best of our knowledge, the interaction mechanism of BSA and SPIONs is still poorly understood. FTIR confirmed the BSA coverage on T-SPIONs and C-SPIONs surfaces and their interaction.. BSA-TSPIONs and BSA-C-SPIONs were separated from the excess BSA solution by centrifugation at $10000 \mathrm{rcf}$ for 3h (under which condition excess BSA have been proved not to be centrifuged down (Casals et al. 2010)), dried completely and then FTIR spectra were collected. Spectra of BSA-T-SPIONs and BSA-C-SPIONs show clearly two adsorption bands at wavelength around $1635 \mathrm{~cm}^{-1}$ and $1515 \mathrm{~cm}^{-1}$ (Fig. 2A-d and Fig. 2B-d), which are characteristic of amide I and amide II bonds of proteins, further confirming the formation of BSA layer on the surface of BSA-T-SPIONs and BSA-C-SPIONs. Moreover, FTIR spectrum could help us reveal the binding mechanism of BSA onto SPIONs surface. FTIR spectrum of BSA-T-SPIONs (Fig. 2A-d) shows an adsorption band at $975 \mathrm{~cm}^{-1}$, this band is assigned to the asymmetric methyl deformation mode C-N on TMAOH (Ouasri et al. 2002), though with $30 \mathrm{~nm}$ shift from $944 \mathrm{~cm}^{-1}$. Another band also appears at $1394 \mathrm{~cm}^{-1}$ (Fig. 2A-d), characteristic of the asymmetric methyl deformation mode of $-\left(\mathrm{CH}_{3}\right)$ on TMAOH (Andrade et al. 2012). These results indicate that TMAOH ligands still exist on BSA-T-SPIONs, implying that the binding of BSA to TSPIONs surface is through interactions with the TMAOH layer, instead of replacing it. The FTIR spectrum of BSA-C-SPIONs present an absorption band at $1450 \mathrm{~cm}^{-1}$ (shifts from $1415 \mathrm{~cm}^{-1}$ Fig. 2B-d), characteristic of asymmetric stretches of $\mathrm{RCO}_{2}$ of $\mathrm{Na}_{3} \mathrm{Cit}$ (Sasidharan et al. 2013), suggesting the existence of citrate ligands on BSA-C-SPIONs. The disappearance of absorption bands at $1579 \mathrm{~cm}^{-1}$ of $\mathrm{Na}_{3} \mathrm{Cit}$ and band shift from $1415 \mathrm{~cm}^{-1}$ to $1450 \mathrm{~cm}^{-1}$ imply strong interaction of citrate group with BSA. 
Therefore, we concluded that interaction between BSA and surface coating of SPIONs results in the formation of BSA monolayer on SPIONs. The additional BSA monolayer maintains the electrostatic repulsion force and the steric hindrance between each SPIONs and hence protecting them from aggregating in biological media.

\section{Conclusion}

The present work provides a facile, rapid and cost effective microwave assisted method to synthesize CSPIONs with robust stability in biological media for the first time. Although with an identical SPION core, the distinct behavior of T-SPIONs and C-SPIONs in biological media implies that surface coating of SPIONs plays a very important role in determining their stability.

The use of BSA showed great efficiency in enhancing the stability of both T-SPIONs and C-SPIONs in several biological media, while the addition of excess $\mathrm{Na}_{3} \mathrm{Cit}$ was only suitable for C-SPIONs. The necessary BSA concentration to provide sufficient stability to both SPIONs in biological media depended on the nature of the tested media, as well as the surface coating of SPIONs. FTIR, Cryo TEM and negative staining TEM reveal that BSA binds to T-SPIONs and C-SPIONs by interacting with TMAOH and citrate coating, forming a BSA monolayer with a thickness of about $3 \pm 1 \mathrm{~nm}$ on both SPIONs. Likely, the additional BSA monolayer preserves the electrostatic repulsion force among the SPIONs while increasing the steric hindrance between SPIONs, preventing their aggregation in biological media.

This work provides a reference for preparing stable SPIONs dispersion used for biological applications, which could lead to the development of reliable in vitro SPIONs toxicity assessments and accurate evaluation of SPIONs dosage needed for in vivo treatment.

\section{Electronic supplementary material}

The online version of this article contains supplementary material, which is available to authorized users.

\section{Acknowledgements}

The research leading to these results has received funding from the People Program (Marie Curie Actions) of the European Union's Seventh Framework Program (FP7/2007-2013) under REA grant agreement $n^{\circ} 303630$ and cofounded by the European Social Fund. Authors acknowledge the funding from Spanish Ministry of Economy MAT 2012-35324, COST Action MP1202 and Ramon y Cajal grant RYC-2010-06082 (AL), China Scholarship Council fellowship (SMY, 201206150053). 


\section{References}

Allouni ZE, Cimpan MR, Høl PJ, Skodvin T, Gjerdet NR (2009) Agglomeration and sedimentation of TiO2 nanoparticles in cell culture medium. Colloid Surface B 68 (1):83-87. doi:10.1016/j.colsurfb.2008.09.014

Andrade ÂL, Valente MA, Ferreira JMF, Fabris JD (2012) Preparation of size-controlled nanoparticles of magnetite. J Magn Magn Mater 324 (10):1753-1757. doi:10.1016/j.jmmm.2011.12.033

Baghbanzadeh M, Carbone L, Cozzoli PD, Kappe CO (2011) Microwave-Assisted Synthesis of Colloidal Inorganic Nanocrystals. Angew Chem Int Edit 50 (48):11312-11359. doi:10.1002/anie.201101274

Brewer SH, Glomm WR, Johnson MC, Knag MK, Franzen S (2005) Probing BSA Binding to Citrate-Coated Gold Nanoparticles and Surfaces. Langmuir 21 (20):9303-9307. doi:10.1021/la050588t

Butoescu N, Jordan O, Burdet P, Stadelmann P, Petri-Fink A, Hofmann H, Doelker E (2009) Dexamethasonecontaining biodegradable superparamagnetic microparticles for intra-articular administration: Physicochemical and magnetic properties, in vitro and in vivo drug release. Eur J Pharm Biopharm 72 (3):529-538. doi:10.1016/j.ejpb.2009.03.003

Carenza E, Barceló V, Morancho A, Levander L, Boada C, Laromaine A, Roig A, Montaner J, Rosell A (2014a) In vitro angiogenic performance and in vivo brain targeting of magnetized endothelial progenitor cells for neurorepair therapies. Nanomed-Nanotechnol 10 (1):225-234. doi:10.1016/j.nano.2013.06.005

Carenza E, Barceló V, Morancho A, Montaner J, Rosell A, Roig A (2014b) Fast synthesis of water-dispersable SPIONs by microwave assisted route for safe labeling of endothelial progenitor cells. Acta Biomater. doi:DOI: 10.1016/j.actbio.2014.04.010

Casals E, Pfaller T, Duschl A, Oostingh GJ, Puntes V (2010) Time Evolution of the Nanoparticle Protein Corona. ACS Nano 4 (7):3623-3632. doi:10.1021/nn901372t

Corti M, Lascialfari A, Marinone M, Masotti A, Micotti E, Orsini F, Ortaggi G, Poletti G, Innocenti C, Sangregorio C (2008a) Magnetic and relaxometric properties of polyethylenimine-coated superparamagnetic MRI contrast agents. J Magn Magn Mater 320 (14):e316-e319. doi:10.1016/j.jmmm.2008.02.115

Corti M, Lascialfari A, Micotti E, Castellano A, Donativi M, Quarta A, Cozzoli PD, Manna L, Pellegrino T, Sangregorio C (2008b) Magnetic properties of novel superparamagnetic MRI contrast agents based on $\begin{array}{llllll}\text { colloidal nanocrystals. J Magn } & \text { Magn } & \text { Mater } 320 \text {-e323. }\end{array}$ doi:http://dx.doi.org/10.1016/j.jmmm.2008.02.064

Deng Y, Wang L, Yang W, Fu S, Elaïssari A (2003) Preparation of magnetic polymeric particles via inverse microemulsion polymerization process. J Magn Magn Mater 257 (1):69-78. doi:10.1016/S03048853(02)00987-3

Di Corato R, Piacenza P, Musarò M, Buonsanti R, Cozzoli PD, Zambianchi M, Barbarella G, Cingolani R, Manna L, Pellegrino T (2009) Magnetic-Fluorescent Colloidal Nanobeads: Preparation and Exploitation in Cell Separation Experiments. Macromol Biosci 9 (10):952-958. doi:10.1002/mabi.200900154

Dominguez-Medina S, Blankenburg J, Olson J, Landes CF, Link S (2013) Adsorption of a Protein Monolayer via Hydrophobic Interactions Prevents Nanoparticle Aggregation under Harsh Environmental Conditions. ACS Sustainable Chem Eng 1 (7):833-842. doi:10.1021/sc400042h

Eberbeck D, Kettering M, Bergemann C, Zirpel P, Hilger I, Trahms L (2010) Quantification of the aggregation of magnetic nanoparticles with different polymeric coatings in cell culture medium. J Phys D Appl Phys 43 (40). doi:10.1088/0022-3727/43/40/405002

Euliss LE, Grancharov SG, O'Brien S, Deming TJ, Stucky GD, Murray CB, Held GA (2003) Cooperative 
Assembly of Magnetic Nanoparticles and Block Copolypeptides in Aqueous Media. Nano Lett 3 (11):1489-1493. doi:10.1021/n1034472y

Figuerola A, Fiore A, Di Corato R, Falqui A, Giannini C, Micotti E, Lascialfari A, Corti M, Cingolani R, Pellegrino T, Cozzoli PD, Manna L (2008) One-Pot Synthesis and Characterization of Size-Controlled Bimagnetic FePt-Iron Oxide Heterodimer Nanocrystals. J Am Chem Soc 130 (4):1477-1487. doi:10.1021/ja078034v

Freese C, Uboldi C, Gibson M, Unger R, Weksler B, Romero I, Couraud P-O, Kirkpatrick C (2012) Uptake and cytotoxicity of citrate-coated gold nanospheres: Comparative studies on human endothelial and epithelial cells. Part Fibre Toxicol 9 (1):23. doi:10.1186/1743-8977-9-23

Geppert M, Petters C, Thiel K, Dringen R (2012) The presence of serum alters the properties of iron oxide nanoparticles and lowers their accumulation by cultured brain astrocytes. J Nanopart Res 15 (1):1-15. doi:10.1007/s11051-012-1349-8

Grief AD, Richardson G (2005) Mathematical modelling of magnetically targeted drug delivery. J Magn Magn Mater 293 (1):455-463. doi:10.1016/j.jmmm.2005.02.040

Guiot C, Spalla O (2012) Stabilization of TiO2 Nanoparticles in Complex Medium through a pH Adjustment Protocol. Environ Sci Technol 47 (2):1057-1064. doi:10.1021/es3040736

Gupta AK, Gupta M (2005) Synthesis and surface engineering of iron oxide nanoparticles for biomedical applications. Biomaterials 26 (18):3995-4021. doi:10.1016/j.biomaterials.2004.10.012

He XM, Carter DC (1992) Atomic structure and chemistry of human serum albumin. Nature 358 (6383):209215. doi:10.1038/358209a0

Hergt R, Dutz S (2007) Magnetic particle hyperthermia—biophysical limitations of a visionary tumour therapy. J Magn Magn Mater 311 (1):187-192. doi:10.1016/j.jmmm.2006.10.1156

Hondow N, Brydson R, Wang P, Holton M, Brown MR, Rees P, Summers H, Brown A (2012) Quantitative characterization of nanoparticle agglomeration within biological media. J Nanopart Res 14 (7):1-15. doi:10.1007/s11051-012-0977-3

Hoskins C, Wang L, Cheng W, Cuschieri A (2012) Dilemmas in the reliable estimation of the in-vitro cell viability in magnetic nanoparticle engineering: which tests and what protocols? Nanoscale Res Lett 7 (1):77. doi:10.1186/1556-276X-7-77

Janes KA, Calvo P, Alonso MJ (2001) Polysaccharide colloidal particles as delivery systems for macromolecules. Adv Drug Deliver Rev 47 (1):83-97. doi:10.1016/S0169-409X(00)00123-X

Ji Z, Jin X, George S, Xia T, Meng H, Wang X, Suarez E, Zhang H, Hoek EMV, Godwin H, Nel AE, Zink JI (2010) Dispersion and Stability Optimization of TiO2 Nanoparticles in Cell Culture Media. Environ Sci Technol 44 (19):7309-7314. doi:10.1021/es100417s

Kah JCY, Chen J, Zubieta A, Hamad-Schifferli K (2012) Exploiting the Protein Corona around Gold Nanorods for Loading and Triggered Release. ACS Nano 6 (8):6730-6740. doi:10.1021/nn301389c

Khullar P, Singh V, Mahal A, Dave PN, Thakur S, Kaur G, Singh J, Singh Kamboj S, Singh Bakshi M (2012) Bovine Serum Albumin Bioconjugated Gold Nanoparticles: Synthesis, Hemolysis, and Cytotoxicity toward Cancer Cell Lines. J Phys Chem C 116 (15):8834-8843. doi:10.1021/jp300585d

Kim DK, Zhang Y, Kehr J, Klason T, Bjelke B, Muhammed M (2001) Characterization and MRI study of surfactant-coated superparamagnetic nanoparticles administered into the rat brain. J Magn Magn Mater 225 (1-2):256-261. doi:10.1016/S0304-8853(00)01255-5

Kircheis R, Wightman L, Wagner E (2001) Design and gene delivery activity of modified polyethylenimines. Adv Drug Deliver Rev 53 (3):341-358. doi:10.1016/S0169-409X(01)00202-2

Kohli I, Alam S, Patel B, Mukhopadhyay A (2013) Interaction and diffusion of gold nanoparticles in bovine 
serum albumin solutions. Appl Phys Lett 102 (20):203705-203705-203704. doi:10.1063/1.4807672

Lee P, Knight R, Smit JM, Wilschut J, Griffin DE (2002) A Single Mutation in the E2 Glycoprotein Important for Neurovirulence Influences Binding of Sindbis Virus to Neuroblastoma Cells. J Virol 76 (12):63026310. doi:10.1128/jvi.76.12.6302-631-.2002

Levy M, Quarta A, Espinosa A, Figuerola A, Wilhelm C, García-Hernández M, Genovese A, Falqui A, Alloyeau D, Buonsanti R, Cozzoli PD, García MA, Gazeau F, Pellegrino T (2011) Correlating Magneto-Structural Properties to Hyperthermia Performance of Highly Monodisperse Iron Oxide Nanoparticles Prepared by a Seeded-Growth Route. Chem Mater 23 (18):4170-4180. doi:10.1021/cm201078f

Liu G, Hong RY, Guo L, Li YG, Li HZ (2011) Preparation, characterization and MRI application of carboxymethyl dextran coated magnetic nanoparticles. Appl Surf Sci 257 (15):6711-6717. doi:10.1016/j.apsusc.2011.02.110

Lu A-H, Salabas EL, Schüth F (2007) Magnetic Nanoparticles: Synthesis, Protection, Functionalization, and Application. Angew Chem Int Edit 46 (8):1222-1244. doi:10.1002/anie.200602866

Luciani N, Gazeau F, Wilhelm C (2009) Reactivity of the monocyte/macrophage system to superparamagnetic anionic nanoparticles. J Mater Chem 19 (35):6373-6380. doi:10.1039/B903306H

McBain SC, Griesenbach U, Xenariou S, Keramane A, Batich CD, Alton EWFW, Dobson J (2008) Magnetic nanoparticles as gene delivery agents: enhanced transfection in the presence of oscillating magnet arrays. Nanotechnology 19 (40). doi:10.1088/0957-4484/19/40/405102

Metin C, Lake L, Miranda C, Nguyen Q (2011) Stability of aqueous silica nanoparticle dispersions. J Nanopart Res 13 (2):839-850. doi:10.1007/s11051-010-0085-1

Moulin AM, O'Shea SJ, Badley RA, Doyle P, Welland ME (1999) Measuring Surface-Induced Conformational Changes in Proteins. Langmuir 15 (26):8776-8779. doi:10.1021/la990416u

Nakata S, Kido N, Hayashi M, Hara M, Sasabe H, Sugawara T, Matsuda T (1996) Chemisorption of proteins and their thiol derivatives onto gold surfaces: characterization based on electrochemical nonlinearity. Biophys Chem 62 (1-3):63-72. doi:10.1016/S0301-4622(96)02208-9

Namduri H, Nasrazadani S (2008) Quantitative analysis of iron oxides using Fourier transform infrared spectrophotometry. Corros Sci 50 (9):2493-2497. doi:10.1016/j.corsci.2008.06.034

Natte K, Friedrich JF, Wohlrab S, Lutzki J, von Klitzing R, Österle W, Orts-Gil G (2013) Impact of polymer shell on the formation and time evolution of nanoparticle-protein corona. Colloid Surface B 104 (0):213-220. doi:10.1016/j.colsurfb.2012.11.019

Nigam S, Barick KC, Bahadur D (2011) Development of citrate-stabilized Fe3O4 nanoparticles: Conjugation and release of doxorubicin for therapeutic applications. J Magn Magn Mater 323 (2):237-243. doi:10.1016/j.jmmm.2010.09.009

Ouasri A, Rhandour A, Dhamelincourt MC, Dhamelincourt P, Mazzah A (2002) Vibrational study of (CH3)4NSbCl6 and [(CH3)4N]2SiF6. Spectrochim Acta A 58 (12):2779-2788. doi:10.1016/S13861425(02)00019-7

Pamme N, Wilhelm C (2006) Continuous sorting of magnetic cells via on-chip free-flow magnetophoresis. Lab Chip 6 (8):974-980. doi:10.1039/B604542A

Pascu O, Carenza E, Gich M, Estradé S, Peiró F, Herranz G, Roig A (2012) Surface Reactivity of Iron Oxide Nanoparticles by Microwave-Assisted Synthesis; Comparison with the Thermal Decomposition Route. J Phys Chem C 116 (28):15108-15116. doi:10.1021/jp303204d

Petri-Fink A, Steitz B, Finka A, Salaklang J, Hofmann H (2008) Effect of cell media on polymer coated superparamagnetic iron oxide nanoparticles (SPIONs): Colloidal stability, cytotoxicity, and cellular 
uptake studies. Eur J Pharm Biopharm 68 (1):129-137. doi:10.1016/j.ejpb.2007.02.024

Porter D, Sriram K, Wolfarth M, Jefferson A, Schwegler-Berry D, Andrew ME, Castranova V (2008) A biocompatible medium for nanoparticle dispersion. Nanotoxicology 2 (3):144-154. doi: $10.1080 / 17435390802318349$

Pratten MK, Lloyd JB (1986) Pinocytosis and phagocytosis: the effect of size of a particulate substrate on its mode of capture by rat peritoneal macrophages cultured in vitro. BBA-Gen Subjects 881 (3):307-313. doi:10.1016/0304-4165(86)90020-6

Safi M, Courtois J, Seigneuret M, Conjeaud H, Berret JF (2011) The effects of aggregation and protein corona on the cellular internalization of iron oxide nanoparticles. Biomaterials 32 (35):9353-9363. doi:10.1016/j.biomaterials.2011.08.048

Safi M, Sarrouj H, Sandre O, Mignet N, Berret JF (2010) Interactions between sub-10-nm iron and cerium oxide nanoparticles and $3 \mathrm{~T} 3$ fibroblasts: the role of the coating and aggregation state. Biomaterials 21 (14). doi:10.1088/0957-4484/21/14/145103

Sager TM, Porter DW, Robinson VA, Lindsley WG, Schwegler-Berry DE, Castranova V (2007) Improved method to disperse nanoparticles for in vitro and in vivo investigation of toxicity. Nanotoxicology 1 (2):118-129. doi:10.1080/17435390701381596

Sasidharan S, Jayasree A, Fazal S, Koyakutty M, Nair SV, Menon D (2013) Ambient temperature synthesis of citrate stabilized and biofunctionalized, fluorescent calcium fluoride nanocrystals for targeted labeling of cancer cells. Biomater Sci 1 (3):294-305. doi:10.1039/C2BM00127F

Sen T, Mandal S, Haldar S, Chattopadhyay K, Patra A (2011) Interaction of Gold Nanoparticle with Human Serum Albumin (HSA) Protein Using Surface Energy Transfer. 115 (49):24037-24044. doi:10.1021/jp207374g

Soenen SJH, Himmelreich U, Nuytten N, De Cuyper M (2011) Cytotoxic effects of iron oxide nanoparticles and implications for safety in cell labelling. Biomaterials 32 (1):195-205. doi:10.1016/j.biomaterials.2010.08.075

Sun S, Zeng H (2002) Size-Controlled Synthesis of Magnetite Nanoparticles. J Am Chem Soc 124 (28):82048205. doi:10.1021/ja026501x

Uboldi C, Bonacchi D, Lorenzi G, Hermanns MI, Pohl C, Baldi G, Unger R, Kirkpatrick CJ (2009) Gold nanoparticles induce cytotoxicity in the alveolar type-II cell lines A549 and NCIH441. Part Fibre Toxicol 6 (1):18. doi:10.1186/1743-8977-6-18

Walczyk D, Bombelli FB, Monopoli MP, Lynch I, Dawson KA (2010) What the Cell "Sees" in Bionanoscience. J Am Chem Soc 132 (16):5761-5768. doi:10.1021/ja910675v

Wei X, Wei Z, Zhang L, Liu Y, He D (2011) Highly water-soluble nanocrystal powders of magnetite and maghemite coated with gluconic acid: Preparation, structure characterization, and surface coordination. J Colloid Interf Sci 354 (1):76-81. doi:10.1016/j.jcis.2010.10.049

Wells MA, Abid A, Kennedy IM, Barakat AI (2012) Serum proteins prevent aggregation of Fe2O3 and ZnO nanoparticles. Nanotoxicology 6 (8):837-846. doi:10.3109/17435390.2011.625131

Wiogo HTR, Lim M, Bulmus V, Gutiérrez L, Woodward RC, Amal R (2012) Insight into Serum Protein Interactions with Functionalized Magnetic Nanoparticles in Biological Media. Langmuir 28 (9):43464356. doi:10.1021/la204740t

Ye A, Flanagan J, Singh H (2006) Formation of stable nanoparticles via electrostatic complexation between sodium caseinate and gum arabic. Biopolymers 82 (2):121-133. doi:10.1002/bip.20465 
Figure Legends:

Fig. 1 (a) TEM images, (b) particle size histogram, (c) electron diffraction and (d) DLS intensity-weighted size distribution of T-SPIONs and C-SPIONs.

Fig. 2 (A) FTIR spectrum of (a) TMAOH, (b) BSA, (c) T-SPIONs and (d) BSA-T-SPIONs; (B) FTIR spectrum of (a) $\mathrm{Na}_{3} \mathrm{Cit}$, (b) BSA, (c) C-SPIONs and (d) BSA-C-SPIONs.

Fig. 3 Stability of (a) T-SPIONs and (b) C-SPIONs in $\mathrm{H}_{2} \mathrm{O}$, PBS, RPMI-FBS and DMEM-FBS in terms of Dh as a function of time.

Fig. 4 (a) DLS intensity-weighted size distributions of C-SPIONs (control) and the prepared BSA-C-SPIONs at an evaluated time of $1 \mathrm{~h}, 4 \mathrm{~h}, 6 \mathrm{~h}$, and $24 \mathrm{~h}$ in water; (b) Cryo-TEM image of the prepared BSA-CSPIONs after $24 \mathrm{~h}$ preparation.

Fig. 5 DLS intensity-weighted size distributions of C-SPIONs (control) and BSA-C-SPIONs in (a) PBS; (b) RPMI; (c) DMEM; (d) RPMI-FBS and (e) DMEM-FBS at an evaluated time of 1h, 4h, 6h, and $24 \mathrm{~h}$.

Fig. 6 The changes in hydrodynamic size of C-SPIONs in PBS-Na ${ }_{3}$ Cit, RPMI-Na ${ }_{3}$ Cit, DMEM-Na 3 Cit, RPMIFBS-Na 3 Cit and DMEM-FBS-Na ${ }_{3} \mathrm{Cit}$ at an evaluated time of $24 \mathrm{~h}$.

Fig. 7 (a) Hydrodynamic size and (b) zeta potential of T-SPIONs, BSA-T-SPIONs, C-SPIONs and BSA-CSPIONs; negative staining TEM images of (c) BSA-T-SPIONs and (d) BSA-C-SPIONs.

Table 1 The necessary concentration of BSA needed for T-SPIONs and C-SPIONs in different media by using the $\mathrm{pH}$ adjusted and BSA adsorption protocol 
Fig. 1 (a) TEM images, (b) particle size histogram, (c) electron diffraction and (d) DLS intensity-weighted size distribution of T-SPIONs and C-SPIONs.

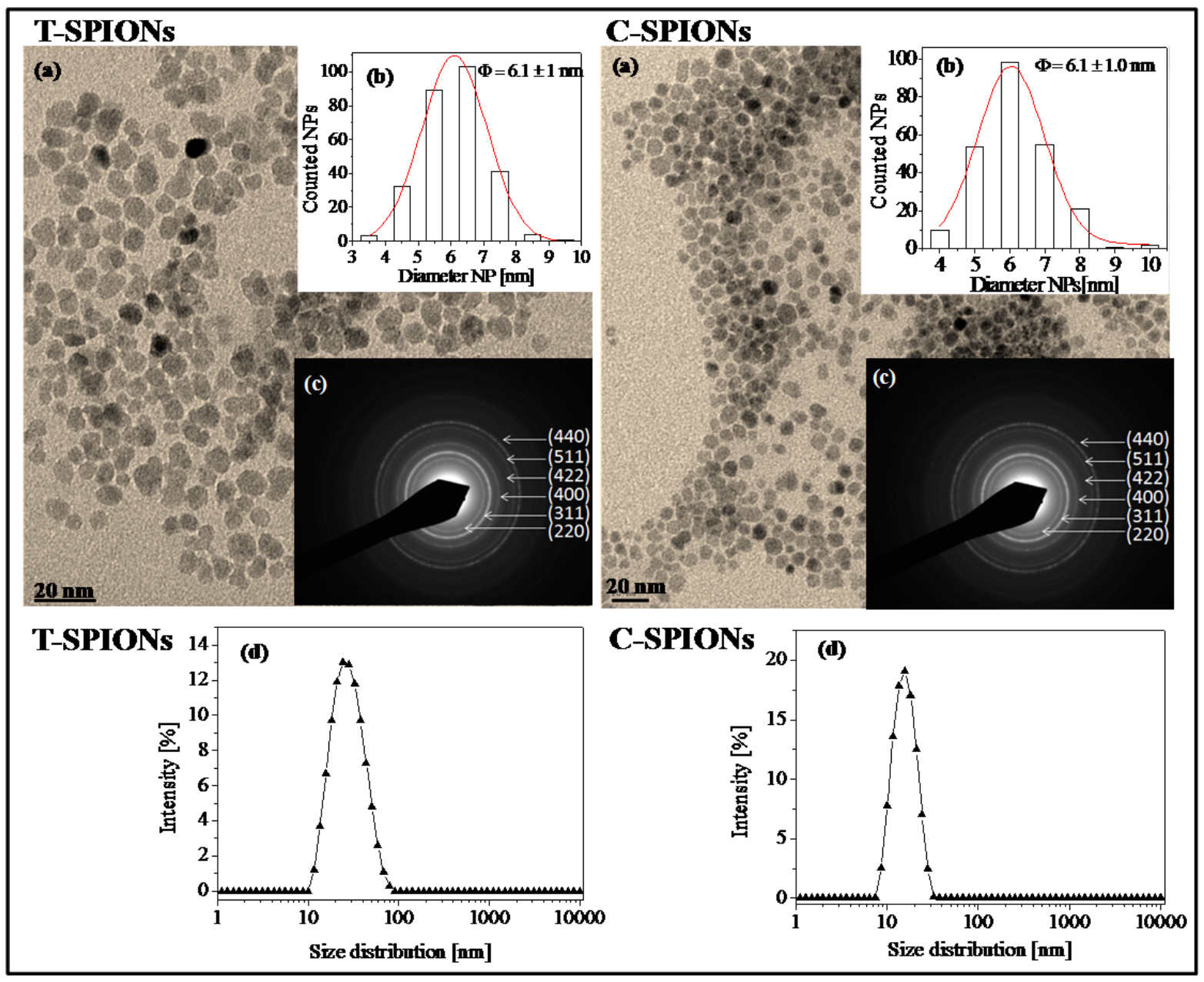


Fig. 2 (A) FTIR spectrum of (a) TMAOH, (b) BSA, (c) T-SPIONs and (d) BSA-T-SPIONs; (B) FTIR spectrum of (a) $\mathrm{Na}_{3} \mathrm{Cit}$, (b) BSA, (c) C-SPIONs and (d) BSA-C-SPIONs.
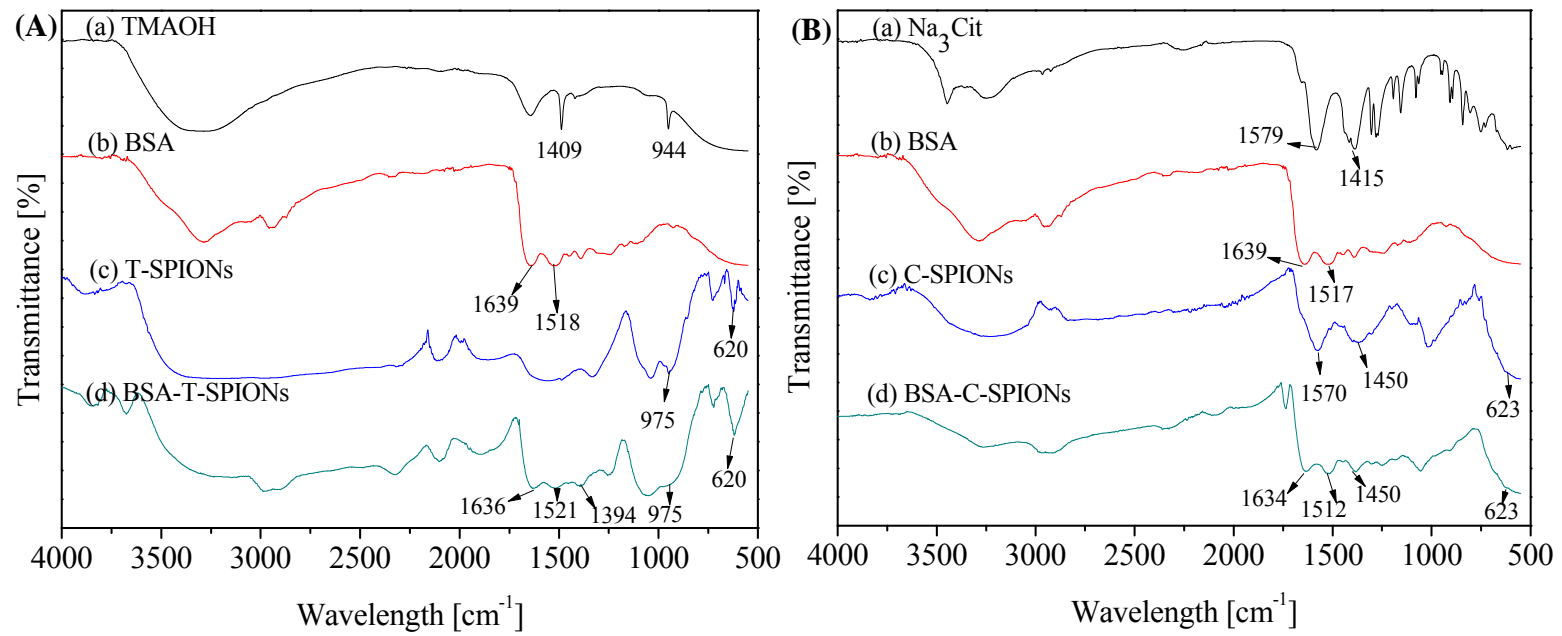
Fig. 3 Stability of (a) T-SPIONs and (b) C-SPIONs in $\mathrm{H}_{2} \mathrm{O}$, PBS, RPMI-FBS and DMEM-FBS in terms of Dh as a function of time.
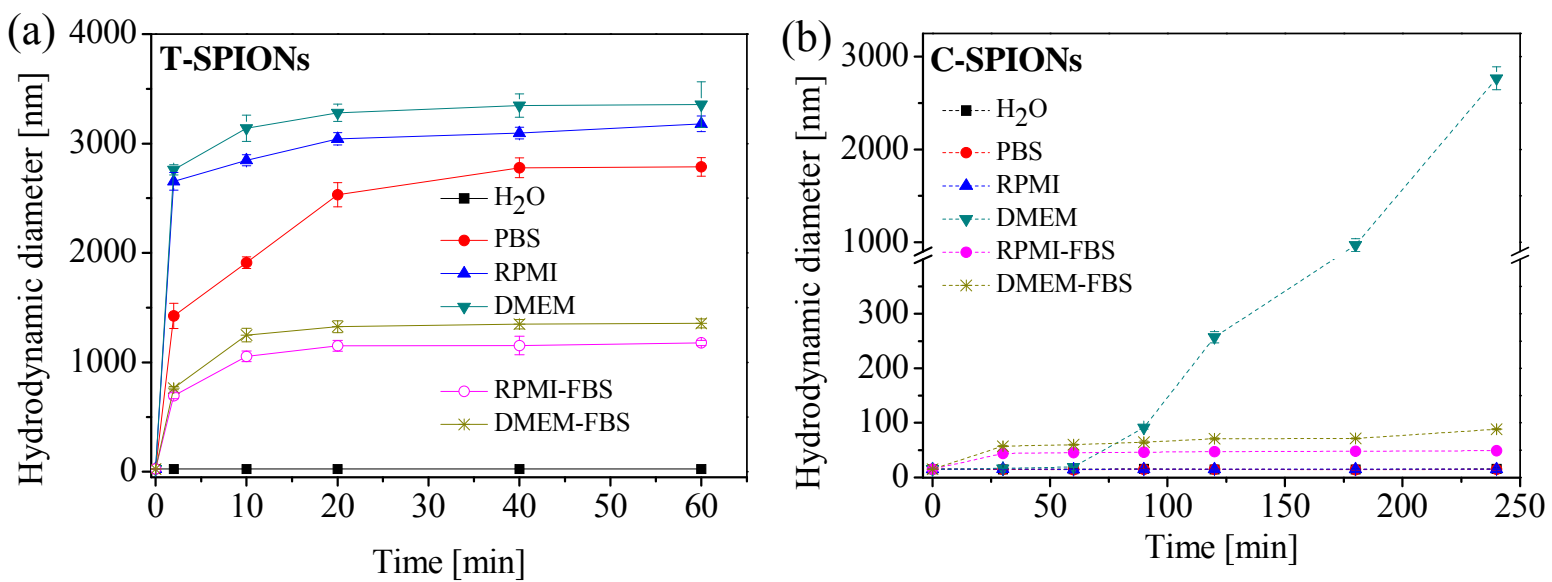
Fig. 4 (a) DLS intensity-weighted size distributions of C-SPIONs (control) and the prepared BSA-C-SPIONs at an evaluated time of $1 \mathrm{~h}, 4 \mathrm{~h}, 6 \mathrm{~h}$, and $24 \mathrm{~h}$ in water; (b) Cryo-TEM image of the prepared BSA-CSPIONs after $24 \mathrm{~h}$ preparation.
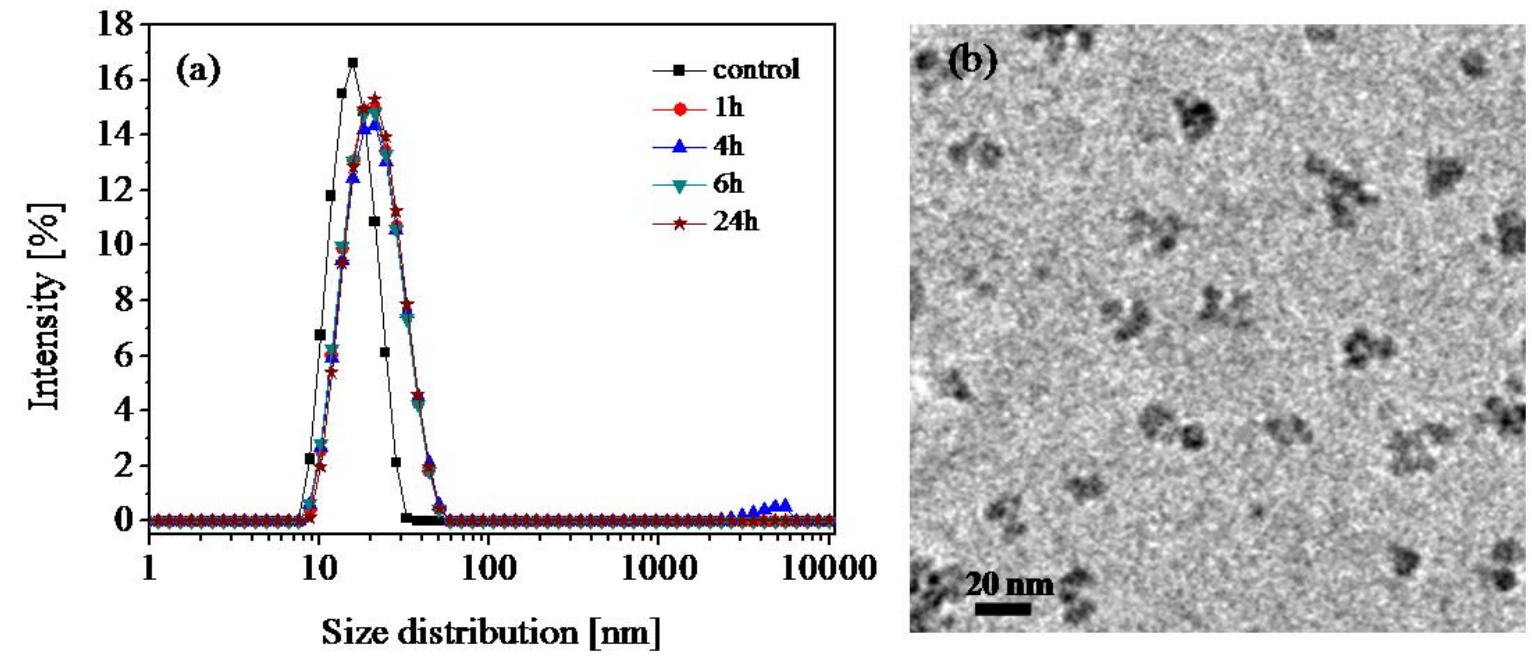
Fig. 5 DLS intensity-weighted size distributions of C-SPIONs (control) and BSA-C-SPIONs in (a) PBS; (b) RPMI; (c) DMEM; (d) RPMI-FBS and (e) DMEM-FBS at an evaluated time of 1h, 4h, 6h, and 24h.
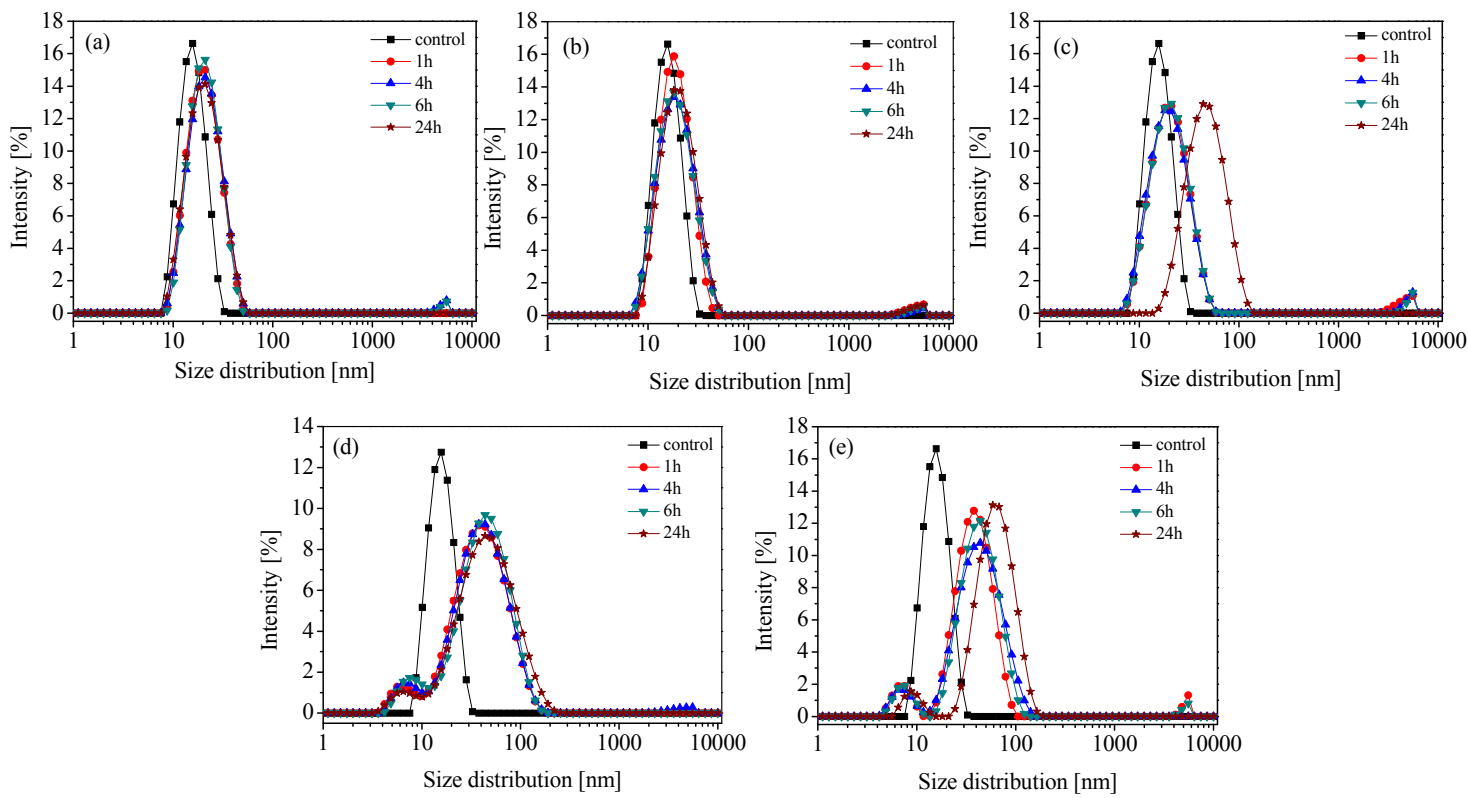
Fig. 6 The changes in hydrodynamic size of C-SPIONs in PBS-Na ${ }_{3}$ Cit, RPMI-Na ${ }_{3}$ Cit, DMEM-Na ${ }_{3}$ Cit, RPMIFBS-Na ${ }_{3} \mathrm{Cit}$ and DMEM-FBS-Na $\mathrm{Cit}_{3}$ at an evaluated time of $24 \mathrm{~h}$.

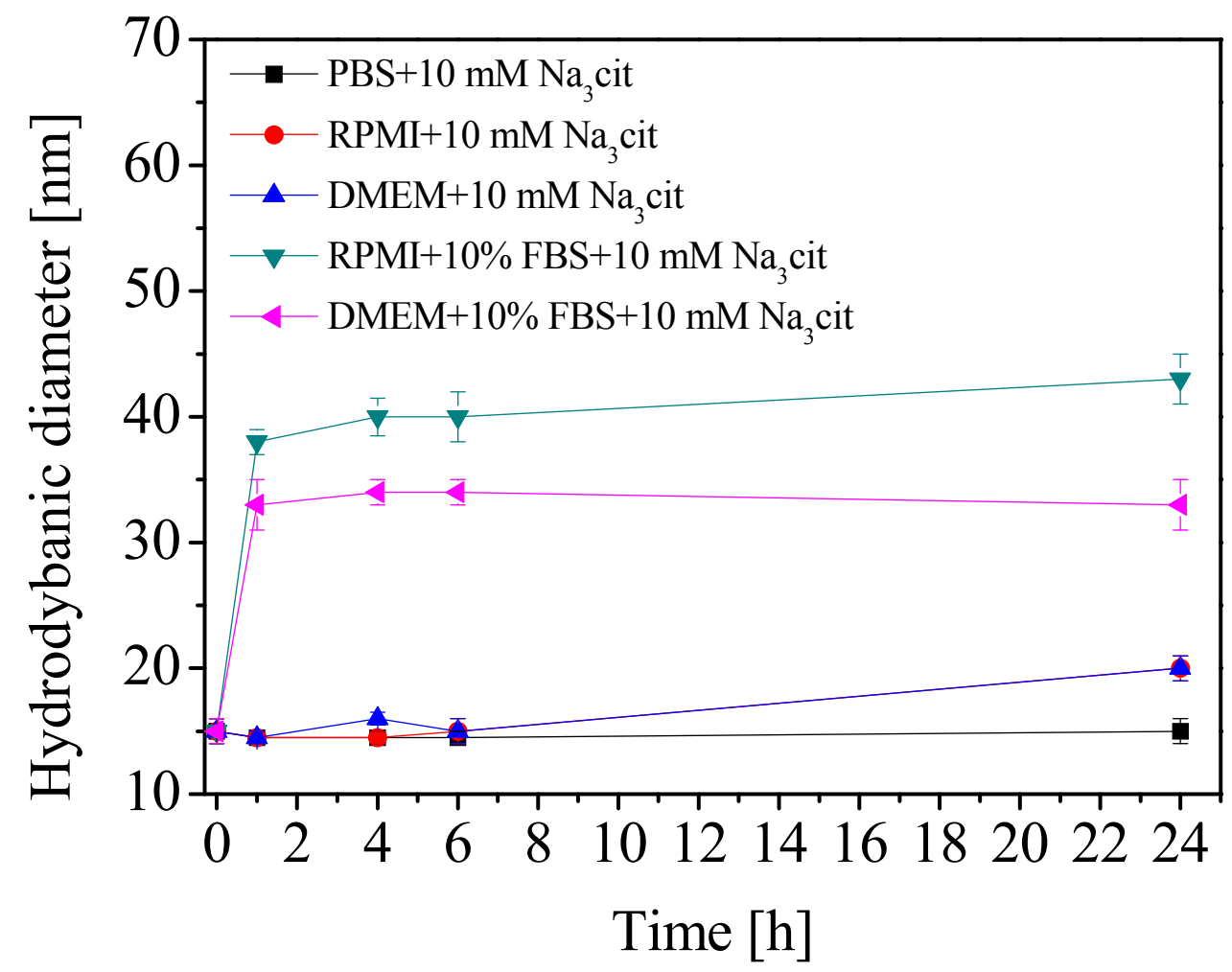


Fig. 7 (a) Hydrodynamic size and (b) zeta potential of T-SPIONs, BSA-T-SPIONs, C-SPIONs and BSA-CSPIONs; negative staining TEM images of (c) BSA-T-SPIONs and (d) BSA-C-SPIONs.
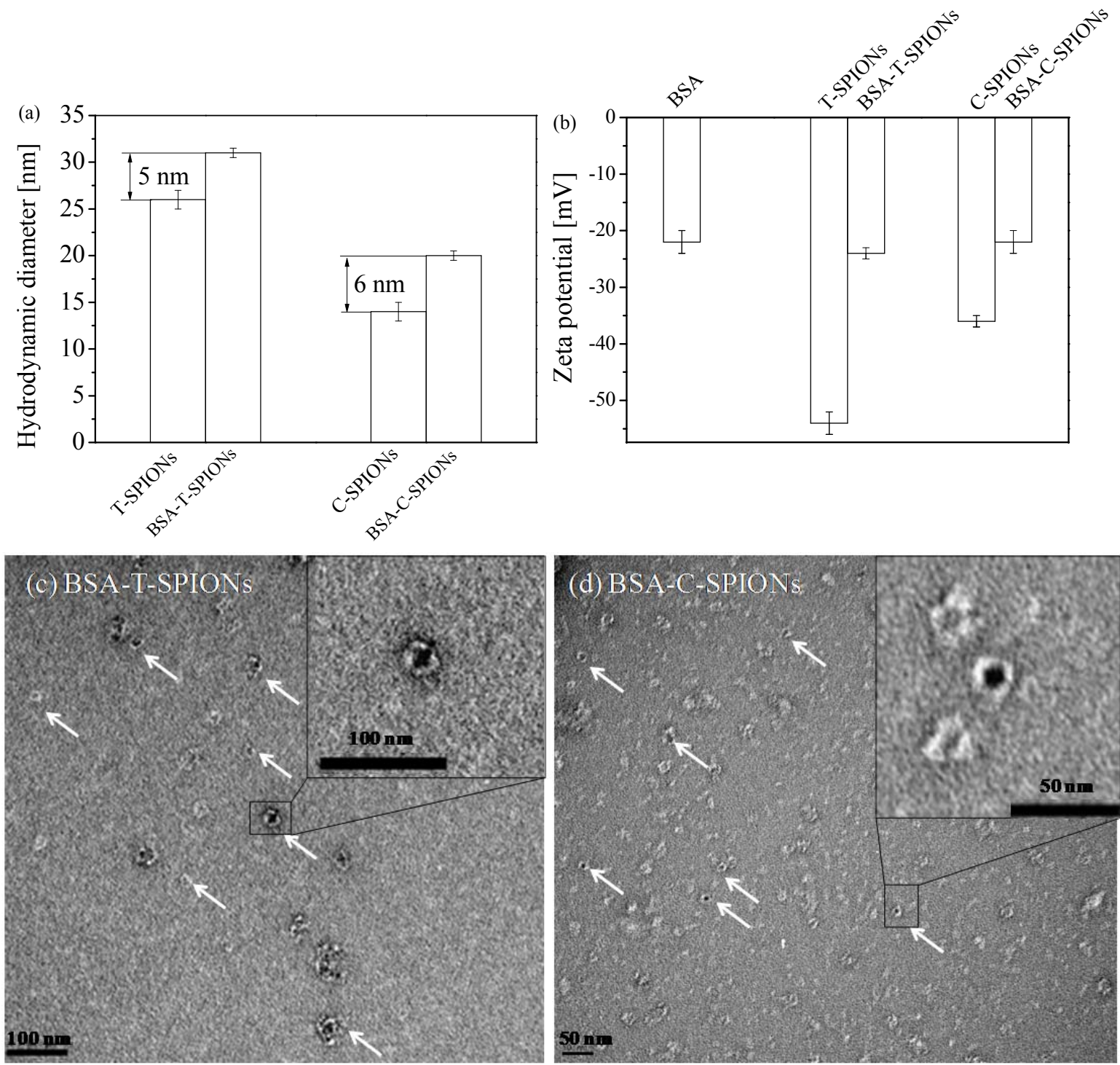
Table 1 The necessary concentration of BSA needed for T-SPIONs and C-SPIONs in different media by using the $\mathrm{pH}$ adjusted and BSA adsorption protocol

\begin{tabular}{lccccc} 
& \multicolumn{5}{c}{ Concentration of BSA needed (w/v \%) } \\
\cline { 2 - 5 } SPIONs & PBS & RPMI & DMEM & RPMI-FBS & DMEM-FBS \\
& 2 & 10 & 15 & 1 & 1 \\
T-SPIONs & 1 & 2 & 5 & 0.5 & 0.5 \\
C-SPIONs & & & & \\
\hline
\end{tabular}

